\title{
Ideal structure and factorization properties of the regular kernel operators
}

\author{
A. Blanco ${ }^{1}$
}

Received: 14 February 2019 / Accepted: 5 December 2019 / Published online: 18 December 2019

(c) The Author(s) 2019

\begin{abstract}
We consider the structure of the lattice of (order and algebra) ideals of the band of regular kernel operators on $L^{p}$-spaces. We show, in particular, that for any $L^{p}(\mu)$ space, with $\mu \sigma$-finite and $1<p<\infty$, the norm-closure of the ideal of finite-rank operators on $L^{p}(\mu)$, is the only non-trivial proper closed (order and algebra) ideal of this band. Key to our results in the $L^{p}$ setting is the fact that every regular kernel operator on an $L^{p}(\mu)$ space ( $\mu$ and $p$ as before) factors with regular factors through $\ell_{p}$. We show that a similar but weaker factorization property, where $\ell_{p}$ is replaced by some reflexive purely atomic Banach lattice, characterizes the regular kernel operators from a reflexive Banach lattice with weak order unit to a KB-space with weak order unit.
\end{abstract}

Keywords Algebra ideal · Banach lattice $\cdot$ Kernel operator $\cdot$ Order ideal $\cdot$ Regular operator

Mathematics Subject Classification Primary 46B28 - 47B07 - 47L10; Secondary 46B42 - 47B65

\section{Introduction}

Let $X$ be a Banach lattice and let $\mathcal{L}^{r}(X)$ be the space of all regular maps (i.e., linear combinations of positive maps) from $X$ to itself. It is well known that $\mathcal{L}^{r}(X)$, endowed with the regular norm and with composition as product, is a Banach algebra and an ordered vector space whose positive cone is closed under multiplication.

There has been interest in understanding the structure of the lattice of closed ideals of $\mathcal{L}^{r}(X)$, where by ideal, here and henceforth, we shall mean an order and two-sided algebra ideal. For instance, if $X=\ell_{p}(1<p<\infty)$ then $\mathcal{A}^{r}\left(\ell_{p}\right)(:=$ the closure of

\footnotetext{
$凶 \quad$ A. Blanco

a.blanco@qub.ac.uk

1 Mathematical Sciences Research Centre, Queen's University Belfast, Belfast, UK
} 
the ideal of finite-rank operators in $\left.\mathcal{L}^{r}\left(\ell_{p}\right)\right)$ is the only non-trivial proper closed ideal of $\mathcal{L}^{r}\left(\ell_{p}\right)[3,4]$. If $X=c_{0}$ or $\ell_{1}$, then $\mathcal{L}^{r}(X)=\mathcal{B}(X)(:=$ the Banach algebra of all bounded operators on $X$ ) and the same conclusion follows from a classical result of Gohberg, Markus and Feldman. It is also known that if $X=L^{p}[0,1](1<p<\infty)$ then the structure of the lattice of closed ideals of $\mathcal{L}^{r}(X)$ significantly departs from that of the lattice of closed (two-sided) algebra ideals of $\mathcal{B}(X)$, e.g., for every $1<p<\infty$, there are in $\mathcal{L}^{r}\left(L^{p}[0,1]\right)$ at least four distinct well-documented closed ideals apart from the trivial ones, namely, $\mathcal{A}^{r}\left(L^{p}[0,1]\right), \mathcal{I}^{r}\left(L^{p}[0,1]\right)$ (see below for definitions), the span of the positive compact operators and the AM-compact operators (this can be easily deduced, for instance, from [22, Theorem 125.5], [17, Theorem 3.3] and [20, Theorem 3.4]).

In this note, our main concern will be with the band $\mathcal{I}^{r}(X)$ of all kernel (or integral) operators in $\mathcal{L}^{r}(X)$. When $X$ is order continuous, the latter is a closed ideal (in the sense of the note) of $\mathcal{L}^{r}(X)$. If $X$ is in addition atomic then $\mathcal{I}^{r}(X)=\mathcal{L}^{r}(X)$ and one can think of the results of [3,4], mentioned above, as results about the ideal structure of $\mathcal{I}^{r}(X)$. Arendt and Sourour [3] asked whether $\mathcal{A}^{r}\left(L^{2}[0,1]\right)$ is likewise the only non-trivial proper closed ideal of $\mathcal{I}^{r}\left(L^{2}[0,1]\right)$. Here, we answer this question in the affirmative. In fact, we will show that for any $L^{p}(\mu)$ space, with $\mu$ a $\sigma$-finite measure and $1<p<\infty, \mathcal{A}^{r}\left(L^{p}(\mu)\right)$ is the only non-trivial proper closed ideal of $\mathcal{I}^{r}\left(L^{p}(\mu)\right)$.

Key to establishing the result from the previous paragraph will be the fact that the kernel operators in $\mathcal{L}^{r}\left(L^{p}(\mu)\right)$ (with $\mu$ and $p$ as above) are precisely the operators that factor through $\ell_{p}$ with regular factors. It is thus natural to ask whether a similar characterization is possible for arbitrary regular kernel operators. In this direction, our main result will be that a regular operator from a reflexive Banach lattice with a weak order unit into a KB-space also with a weak order unit is a kernel operator if and only if it factors through a reflexive purely atomic Banach lattice with both factors regular. Unfortunately, our path towards this more general but weaker result will be different from the one followed to establish the factorization result in the case of $L^{p}$-spaces. For this reason, it will be discussed separately.

The note has been organized as follows. In the next section we gather some notation and terminology, as well as some basic facts needed throughout the note. In Sect. 3, we first give an extension of the factorization result for regular kernel operators on $L^{p}(\mu)$ spaces, mentioned above, and then prove our main results on the ideal structure of the band of regular kernel operators on such spaces. Whenever possible, we shall work and present our results in a slightly more general framework, which we explain below. Finally, in Sect. 4, we investigate the connection between regular kernel operators and factorization of regular maps (with regular factors) through purely atomic Banach lattices. We prove, amongst other things, the result stated at the end of the previous paragraph.

\section{Some background and notation}

Given a Banach lattice $X$, we shall write $X_{+}$for its positive cone and $X_{[\rho]}$ for the closed ball in $X$ centered at the origin and of radius $\rho$. If $S$ is a subset of $X$, we shall denote by $\operatorname{sp}(S)$ its linear span and by $S^{d d}$ the band in $X$ generated by $S$. In the case 
of an indexed set $\left\{x_{i}: i \in I\right\}$, we shall write $\left[x_{i}: i \in I\right]$ (or just $\left[x_{i}\right]$ if $I$ is clear from context) for the norm-closure of its linear span in $X$.

The topological dual of $X$ will be denoted by $X^{\prime}$, and we shall always identify $X$ with its image in $X^{\prime \prime}$ under the canonical embedding. Recall a Banach lattice $X$ is said to be a KB-space if every increasing norm bounded sequence $\left(x_{n}\right) \subset X$ is norm convergent. Recall also every KB-space is a band in its second dual.

A linear subspace $\mathcal{I}$ of an ordered vector space $V$ shall be called an order ideal if (i) $\pm v \leq w \in \mathcal{I} \Rightarrow v \in \mathcal{I}$; and (ii) for every $v \in \mathcal{I}$ there is $w \in \mathcal{I}$ such that $\pm v \leq w$. One easily checks that, when $V$ is a vector lattice, the latter is equivalent to the standard definition of ideal in the vector lattice setting.

Given a linear map $T: X \rightarrow Y$ and a subspace $E \subset X$ (resp. $F \subset Y$ ), we shall write $\left.T\right|_{E}$ (resp. $\left.T\right|^{F}$ ) for its restriction (resp. corestriction) to $E$ (resp. $F$ ). Moreover, we shall denote by $T^{\prime}$ its (topological) adjoint and by $T(X)$ its image. The identity map on $X$ shall be denoted by $\operatorname{id}_{X}$ (or just id if $X$ is clear from context).

As customary, a linear map $T$ between Banach lattices $X$ and $Y$ shall be said to be positive if $T\left(X_{+}\right) \subseteq Y_{+}$and regular if it can be written as a linear combination of positive maps. We shall write $\mathcal{L}^{r}(X, Y)$ for the linear space of all regular maps from $X$ to $Y$. The latter is an ordered vector space and becomes a Banach space when endowed with the regular norm $\|T\|_{r}:=\{\|S\|: S \in \mathcal{B}(X, Y),|T x| \leq S|x|(x \in X)\}$ $\left(T \in \mathcal{L}^{r}(X, Y)\right)$, where $\|\cdot\|$ stands for the operator norm on $\mathcal{B}(X, Y):=$ the Banach space of all bounded linear operators from $X$ to $Y$. When $Y$ is Dedekind complete, $\mathcal{L}^{r}(X, Y)$ is also a Banach lattice. Given subspaces $\mathcal{I} \subseteq \mathcal{L}^{r}(Y, Z)$ and $\mathcal{J} \subseteq \mathcal{L}^{r}(X, Y)$, we shall write $\mathcal{I} \circ \mathcal{J}$ for the set $\{S T: S \in \mathcal{I}$ and $T \in \mathcal{J}\} \subseteq \mathcal{L}^{r}(X, Z)$.

We shall write $\mathcal{F}(X, Y)$ for the linear space of all continuous finite-rank operators from $X$ to $Y$ and $\mathcal{A}^{r}(X, Y)$ for its norm closure in $\mathcal{L}^{r}(X, Y)$. We shall call the elements of $\mathcal{A}^{r}(X, Y)$ approximable regular operators. Two well-known facts about $\mathcal{A}^{r}(X, Y)$ that we shall use repeatedly later on are that it is always a Banach lattice and that $X_{+}^{\prime} \otimes Y_{+}:=\left\{\sum_{i=1}^{n} x_{i}^{\prime} \otimes y_{i}: x_{i}^{\prime} \in X_{+}^{\prime}, y_{i} \in Y_{+}\right.$and $\left.n \in \mathbb{N}\right\}$ is norm-dense in $\mathcal{A}^{r}(X, Y)_{+}$(here, of course, we are identifying $X^{\prime} \otimes Y$ with $\mathcal{F}(X, Y)$ in the usual way).

Given Banach lattices $X$ and $Y$, we shall say $T \in \mathcal{L}^{r}(X, Y)$ is a regular kernel operator if it belongs to the band $\left(X_{o c}^{\prime} \otimes Y\right)^{d d}$, where we have written $X_{o c}^{\prime}$ for the order continuous dual of $X$, and $X_{o c}^{\prime} \otimes Y$ for the linear space of all order continuous finite-rank operators in $\mathcal{L}^{r}(X, Y)$. It is well known that if $X$ and $Y$ are Banach function spaces on $\sigma$-finite measure spaces $\left(\Omega_{1}, \Sigma_{1}, \mu_{1}\right)$ and $\left(\Omega_{2}, \Sigma_{2}, \mu_{2}\right)$, respectively, then $T \in\left(X_{o c}^{\prime} \otimes Y\right)^{d d}$ if and only if there is a $\mu_{1} \times \mu_{2}$-measurable function on $\Omega_{1} \times \Omega_{2}$ such that $(T f)(x):=\int_{\Omega_{1}} k(t, x) f(t) d \mu_{1}(t)$ for $\mu_{2}$-almost every $x \in \Omega_{2}$ and the function $\int_{\Omega_{1}}|k(t, \cdot)||f(t)| d \mu_{1}(t)$ belongs to $Y(f \in X)$. We shall let $\mathcal{I}^{r}(X, Y):=$ $\left(X_{o c}^{\prime} \otimes Y\right)^{d d}$. When $X$ is order continuous, $X_{o c}^{\prime}=X^{\prime}$ and $\mathcal{I}^{r}(X, Y)=\mathcal{F}(X, Y)^{d d}$. It is known that if $X$ (resp. $Y$ ) is order continuous then for every Banach lattice $E$, $\mathcal{I}^{r}(X, Y) \circ \mathcal{L}^{r}(E, X) \subseteq \mathcal{I}^{r}(E, Y)\left(\operatorname{resp} . \mathcal{L}^{r}(Y, E) \circ \mathcal{I}^{r}(X, Y) \subseteq \mathcal{I}^{r}(X, E)\right)$, and in general, the inclusion may fail. Furthermore, if either $X$ is order continuous and atomic, or if $Y$ is atomic, then $\mathcal{F}(X, Y)^{d d}=\mathcal{L}^{r}(X, Y)$.

A known fact connecting the above classes of regular operators, which we shall use throughout the note, is that if $X^{\prime}$ and $Y$ are order continuous Banach lattices then 
the operators in $\mathcal{I}^{r}(X, Y)$ whose modules are compact are precisely the elements of $\mathcal{A}^{r}(X, Y)$ (see for instance [17, Theorem 3.3]).

To simplify, all Banach lattices of the note will be assumed to be real. Other facts and terminology will be introduced and explained as they are needed. For unexplained material about Banach lattices, we refer the reader to $[2,16]$.

\section{The ideal structure of $\mathcal{I}^{r}\left(L^{p}\right)(1 \leq p<\infty)$}

Mimicking the Banach space situation, given Banach lattices $E, F, X$ and $Y$, and $L \in \mathcal{L}^{r}(E, F)$, we shall say an operator $T \in \mathcal{L}^{r}(X, Y) r$-factors through $L$ if there are operators $R \in \mathcal{L}^{r}(F, Y)$ and $S \in \mathcal{L}^{r}(X, E)$ such that $T=R L S$. We then define

$$
\Gamma_{L, r}(X, Y):=\operatorname{sp}\left\{T \in \mathcal{L}^{r}(X, Y): T r \text {-factors through } L\right\},
$$

and

$$
\Gamma_{L, r}^{\mathrm{o}}(X, Y):=\left\{T \in \mathcal{L}^{r}(X, Y): \pm T \leq S \text { for some } S \in \Gamma_{L, r}(X, Y)\right\}
$$

If $X=Y$ we simply write $\Gamma_{L, r}(X)\left(\operatorname{resp} . \Gamma_{L, r}^{\mathrm{o}}(X)\right)$ for $\Gamma_{L, r}(X, X)\left(\operatorname{resp} . \Gamma_{L, r}^{\mathrm{o}}(X, X)\right)$. Clearly, $\Gamma_{L, r}^{\mathrm{o}}(X, Y)$ is an order ideal of $\mathcal{L}^{r}(X, Y)$ and one can easily verify that for every pair of Banach lattices $E, F, \mathcal{L}^{r}(Y, F) \circ \Gamma_{L, r}(X, Y) \circ \mathcal{L}^{r}(E, X) \subseteq \Gamma_{L, r}(E, F)$ and $\mathcal{L}^{r}(Y, F) \circ \Gamma_{L, r}^{\mathrm{o}}(X, Y) \circ \mathcal{L}^{r}(E, X) \subseteq \Gamma_{L, r}^{\mathrm{o}}(E, F)$. If $L=\mathrm{id}_{Z}$ for some Banach lattice $Z$ then we shall simply say that $T r$-factors through $Z$. (Note the latter simply amounts to $T$ factoring through $Z$, in the usual sense, but with both factors being regular.) In this case, we shall write $\Gamma_{Z, r}$ and $\Gamma_{Z, r}^{\mathrm{o}}$ for $\Gamma_{\mathrm{id}_{Z}, r}$ and $\Gamma_{\mathrm{id}_{Z}, r}^{\mathrm{o}}$, respectively.

Remark 1 In general, $\Gamma_{Z, r}(X, Y) \neq\left\{T \in \mathcal{L}^{r}(X, Y): T r\right.$-factors through $\left.Z\right\}$. However, if $Z$ admits a decomposition $Z_{1} \oplus Z_{2} \oplus Z_{3}$ with $Z_{1}$ and $Z_{2}$ isomorphic as Banach lattices to $Z$ and each 'coordinate' projection $P_{i}: Z \rightarrow Z_{i}(i=1,2,3)$ regular, then equality holds. (The proof of this is the same as in the Banach space situation. Clearly, one only needs to check that the set on the right, call it $\mathcal{V}$, is closed under addition. So let $T_{1}, T_{2} \in \mathcal{V}$ be arbitrary and let $R_{1} S_{1}$ and $R_{2} S_{2}$ be r-factorizations through $Z$ of $T_{1}$ and $T_{2}$, respectively. For each $i \in\{1,2\}$, let $\phi_{i}: Z \rightarrow Z_{i}$ and $\iota_{i}: Z_{i} \rightarrow Z$ be a Banach lattice isomorphism and the corresponding coordinate embedding, respectively, and let $R:=R_{1} \phi_{1}^{-1} P_{1}+R_{2} \phi_{2}^{-1} P_{2}$ and $S:=\iota_{1} \phi_{1} S_{1}+\iota_{2} \phi_{2} S_{2}$. Then $R S=T_{1}+T_{2}$.) The spaces $c_{0}, \ell_{p}$ and $L^{p}[0,1], 1 \leq p \leq \infty$, all have this property.

Remark 2 It may be worth pointing out that, unless $L$ is positive, $\Gamma_{L, r}^{o}(X)$ need not coincide with the order and algebra ideal of $\mathcal{L}^{r}(X)$ generated by $L$. For instance, if $T$ is a compact operator on $\ell_{p}(1<p<\infty)$ whose module is not compact, then there is no $S \in \Gamma_{T, r}\left(\ell_{p}\right)$ such that $|T| \leq S$. One can easily see that the smallest order and algebra ideal of $\mathcal{L}^{r}\left(\ell_{p}\right)$ that contains $T$ is precisely $\Gamma_{|T|, r}^{o}\left(\ell_{p}\right)$. So, in the study of (order and algebra) ideals of $\mathcal{L}^{r}(X), r$-factorization through positive operators has a special significance.

It is known [see [18, Theorems 3.1 and 4.1] (resp. [18, Corollary 3.3])] that if $\left(\Omega_{1}, \Sigma_{1}, \mu_{1}\right)$ and $\left(\Omega_{2}, \Sigma_{2}, \mu_{2}\right)$ are finite measure spaces and $T: L^{p}\left(\mu_{1}\right) \rightarrow L^{p}\left(\mu_{2}\right)$, 
$1<p \leq \infty$ (resp. $1 \leq p<\infty$ ), is a positive kernel operator, then for every $\varepsilon>0$ there is $A \subseteq \Omega_{2}$ (resp. $\left.A \subseteq \Omega_{1}\right)$ of measure $\geq \mu_{2}\left(\Omega_{2}\right)-\varepsilon$ (resp. $\left.\geq \mu_{1}\left(\Omega_{1}\right)-\varepsilon\right)$ such that the map $f \mapsto \chi_{A} T(f), L^{p}\left(\mu_{1}\right) \rightarrow L^{p}\left(\mu_{2}\right)$ (resp. $f \mapsto T\left(\chi_{A} f\right), L^{p}\left(\mu_{1}\right) \rightarrow$ $L^{p}\left(\mu_{2}\right)$ ), is approximable regular. (We should mention here that a similar result to [18, Corollary 3.3] for $p=1$, was first established by Lewis and Stegall in the case where $\mu_{1}$ is a Radon measure and $\Omega_{1}$ is a compact set (see [11, Lemma]).) An easy consequence of these results is that every regular kernel operator $T: L^{p}\left(\mu_{1}\right) \rightarrow$ $L^{p}\left(\mu_{2}\right) r$-factors through $\ell_{p}(1 \leq p \leq \infty)$. The first result of the section will be a slightly more general version of this factorization result in the case where $1<p<\infty$. Recall first a linear operator $T$ from a Banach lattice $X$ to a Banach lattice $Y$ is said to be $p$-convex (resp. $p$-concave) for some $1 \leq p<\infty$ if for some constant $C$ and every finite sequence $x_{1}, \ldots, x_{n} \in X$,

$$
\begin{aligned}
\left\|\left(\sum_{i=1}^{n}\left|T x_{i}\right|^{p}\right)^{\frac{1}{p}}\right\| & \leq C\left(\sum_{i=1}^{n}\left\|x_{i}\right\|^{p}\right)^{\frac{1}{p}}\left(\operatorname{resp} \cdot\left(\sum_{i=1}^{n}\left\|T x_{i}\right\|^{p}\right)^{\frac{1}{p}}\right. \\
& \left.\leq C\left\|\left(\sum_{i=1}^{n}\left|x_{i}\right|^{p}\right)^{\frac{1}{p}}\right\|\right) .
\end{aligned}
$$

In turn, $X$ is said to be $p$-convex (resp. $p$-concave) for some $1 \leq p<\infty$ if $\operatorname{id}_{X}$ is $p$-convex (resp. $p$-concave).

The result on $r$-factorization can now be stated as follows.

Theorem 1 Let $X$ and $Y$ be Banach lattices and let $T \in \mathcal{L}^{r}(X, Y)$. Suppose there is a $\sigma$-finite measure space $(\Omega, \Sigma, \mu)$ such that, for some $1<p<\infty, T r$-factors through $L^{p}(\mu)$ with factors $R: L^{p}(\mu) \rightarrow Y$ and $S: X \rightarrow L^{p}(\mu)$. If $S$ is a regular kernel operator and $X$ is p-convex, or if $R$ is a regular kernel operator and $Y$ is $p$-concave, then $T$-factors through $\ell_{p}$.

Our proof of Theorem 1 shall require the following version of [18, Theorem 3.1].

Lemma 1 Let X be a p-convex Banach lattice, let $(\Omega, \Sigma, \mu)$ be a finite-measure space and let $T: X \rightarrow L^{p}(\mu)(1<p<\infty)$ be a regular kernel operator. Then, for every $\varepsilon>0$ there exists $A \in \Sigma$ such that $\mu(\Omega \backslash A)<\varepsilon$ and $M_{A} \circ T$ is approximable regular, where $M_{A}: L^{p}(\mu) \rightarrow L^{p}(\mu), f \mapsto \chi_{A} f$.

Proof Consider first the case where $T$ is positive. Let $\varepsilon>0$ arbitrary, let $l: L^{p}(\mu) \rightarrow$ $L^{1}(\mu)$ be the natural embedding, and let $\mathcal{T}:=\imath \circ T$, which is also a kernel operator (because $p<\infty)$. Since $X$ is $p$-convex, $s(X)>1$, so $\mathcal{T}$ is approximable regular (see [17, Corollary 3.4(iii)]). Let $\left(U_{n}\right)$ be a sequence in $\mathcal{F}\left(X, L^{p}(\mu)\right)$ such that $\sum_{n} \iota \circ U_{n}=$ $\mathcal{T}$ and $\sum_{n} n\left\|l \circ U_{n}\right\|_{r}<\infty$, and set $\sum_{n} n\left|l \circ U_{n}\right|=: \mathcal{S}: X \rightarrow L^{1}(\mu)$. Since $X$ is $p$-convex and $\mathcal{S} \geq 0, \mathcal{S}$ is $p$-convex too (see [10, Proposition 1.d.9]), and so, it follows from [12, Théorème 2] that there is a strictly positive function $g \in L^{r}(\mu)$ $(r=p /(p-1))$ such that $g^{-1} \mathcal{S}(x) \in L^{p}(\mu)(x \in X)$.

Let $A_{t}:=\{\omega \in \Omega: g(\omega) \geq t\}\left(t \in \mathbb{R}_{+}\right)$and choose $t_{0}$ small enough so that $\mu\left(\Omega \backslash A_{t_{0}}\right)<\varepsilon$. Set $A:=A_{t_{0}}$. It is clear that $\chi_{A} \mathcal{S}(x) \in L^{p}(\mu)(x \in X)$, so $\widetilde{S}$ : 
$X \rightarrow L^{p}(\mu), x \mapsto \chi_{A} \mathcal{S}(x)$, is a well-defined positive (and therefore continuous) operator. To see $M_{A} \circ T: X \rightarrow L^{p}(\mu)$ is regular approximable, first note that $\left(M_{A} \circ T\right)(x)=\sum_{n} \chi_{A} U_{n}(x)(x \in X)$. Then note that, for every $N \in \mathbb{N}$ and $x \in X_{+}$,

$$
\begin{aligned}
\left\|\sum_{n \geq N} \chi_{A} U_{n}(x)\right\| & \leq\left\|\sum_{n \geq N} \chi_{A}\left|\imath \circ U_{n}\right|(x)\right\| \\
& \leq \frac{1}{N}\left\|\sum_{n \geq N} n \chi_{A}\left|\imath \circ U_{n}\right|(x)\right\| \leq \frac{1}{N}\|\widetilde{S}\|\|x\|,
\end{aligned}
$$

so $M_{A} \circ T$ is positive compact. Since $M_{A} \circ T$ is also a kernel operator, it must be approximable regular.

In general, if $T=T_{+}-T_{-}$, then, by the previous part of the argument, for every $\varepsilon>0$ there are sets $A_{1}, A_{2} \in \Sigma$ such that $\mu\left(\Omega \backslash A_{i}\right)<\varepsilon / 2 \quad(i=1,2)$, and both $M_{A_{1}} \circ T_{+}$and $M_{A_{2}} \circ T_{-}$are approximable regular. One then easily verifies that $A:=$ $A_{1} \cap A_{2}$ meets all the requirements.

Proof of Theorem 1 Suppose first $X$ is $p$-convex and $S: X \rightarrow L^{p}(\mu)$ is a positive kernel operator. Clearly, it will suffice to produce an $r$-factorization of $S$ through $\ell_{p}$.

By the previous lemma, there is a partition $\left\{\Omega_{i}: i \in \mathbb{N}\right\}$ of $\Omega$ such that, if $M_{i}$ : $L^{p}(\mu) \rightarrow L^{p}(\mu)$ is defined by $M_{i}(f):=\chi_{\Omega_{i}} f\left(f \in L^{p}(\mu)\right)$, then $M_{i} \circ S: X \rightarrow$ $L^{p}(\mu)$ is approximable regular $(i \in \mathbb{N})$. Let $\varepsilon>0$ arbitrary, and for each $i \in \mathbb{N}$, choose $V_{i}=\sum_{j=1}^{n_{i}} x_{i j}^{\prime} \otimes f_{i j} \in \mathcal{F}\left(X, L^{p}(\mu)\right)_{+}$so that $\left\|V_{i}-M_{i} S\right\|_{r} \leq \varepsilon / 2^{i}$. Let $\widetilde{S}: X \rightarrow L^{p}(\mu)$ be the strong operator limit of the sequence $\left(\sum_{i=1}^{n} V_{i}\right)$.

Since the linear space $S(\mu)$ of all simple integrable functions on $(\Omega, \Sigma, \mu)$ is dense in $L^{p}(\mu)$, we can further assume $\left\{f_{i j}: 1 \leq j \leq n_{i}\right\} \subset M_{i}(S(\mu))(i \in \mathbb{N})$. For each $i$, let $E_{i}$ be the smallest vector sublattice of $L^{p}(\mu)$ such that $\left[f_{i j}: 1 \leq j \leq n_{i}\right] \subseteq E_{i}$, and let $l_{i}: E_{i} \rightarrow L_{p}(\mu)$ be the natural inclusion. Let $E:=\left(\bigoplus_{i} E_{i}\right)_{p}$ (which is clearly isomorphic, as a Banach lattice, to $\left.\ell_{p}\right)$. The maps $S_{2}: X \rightarrow E, x \mapsto\left(V_{i} x\right)$, and $S_{1}: E \rightarrow L^{p}(\mu),\left(z_{i}\right) \mapsto \sum_{i} l_{i}\left(z_{i}\right)$, are both well-defined, for

$$
\begin{aligned}
\left\|S_{2} x\right\|_{E} & =\left(\sum_{i}\left\|V_{i} x\right\|_{E_{i}}^{p}\right)^{1 / p} \\
& \leq\left(\sum_{i}\left\|M_{i} S x\right\|_{E_{i}}^{p}\right)^{1 / p}+\left(\sum_{i}\left\|\left(V_{i}-M_{i} S\right) x\right\|_{E_{i}}^{p}\right)^{1 / p} \\
& \leq\|\widetilde{S} x\|+\varepsilon\left(\sum_{i} 2^{-i p}\right)^{1 / p}\|x\| \leq(\|\widetilde{S}\|+\varepsilon)\|x\| \quad(x \in X),
\end{aligned}
$$

and

$$
\left\|S_{1}\left(\left(z_{i}\right)\right)\right\|_{L^{p}}=\left\|\sum_{i} l_{i}\left(z_{i}\right)\right\|_{L^{p}}=\left(\sum_{i}\left\|l_{i}\left(z_{i}\right)\right\|_{L^{p}}^{p}\right)^{1 / p}=\left\|\left(z_{i}\right)\right\|_{E} \quad\left(\left(z_{i}\right) \in E\right) .
$$

Clearly, they provide an $r$-factorization of $\widetilde{S}$ through $\ell_{p}$ with positive factors. 
To obtain an $r$-factorization of $S$ it just remains to find an $r$-factorization for $S_{0}:=$ $S-\widetilde{S}$. To this end, first choose a sequence $\left(U_{i}\right) \subset \mathcal{F}(X, S(\mu)) \backslash\{0\}$ such that $\sum_{i} U_{i}=$ $S_{0}$ and $\sum_{i}\left\|U_{i}\right\|_{r}=: M<\infty$ (one such sequence exists because $S_{0}=\sum_{i}\left(M_{i} S-\right.$ $\left.\left.V_{i}\right) \in \mathcal{A}^{r}\left(X, L^{p}(\mu)\right)\right)$. For each $i \in \mathbb{N}$, let $F_{i}$ be the smallest Riesz subspace of $L^{p}(\mu)$ that contains $U_{i}(X)$, and let $J_{i}: F_{i} \rightarrow L^{p}(\mu)$ be the natural embedding. Let $F:=\left(\bigoplus_{i} F_{i}\right)_{p}$, let $r_{i}:=\left\|U_{i}\right\|_{r}^{1 / q}(i \in \mathbb{N})$, where $q=p /(p-1)$, let $S_{02}: X \rightarrow F$, $x \mapsto\left(r_{i}^{-1} U_{i}(x)\right)$, and let $S_{01}: F \rightarrow L^{p}(\mu),\left(z_{i}\right) \mapsto \sum_{i} r_{i} J_{i}\left(z_{i}\right)$. Then

$$
\begin{aligned}
\left\|S_{02} x\right\|_{F} & =\left(\sum_{i} r_{i}^{-p}\left\|U_{i} x\right\|_{F_{i}}^{p}\right)^{1 / p} \leq M^{1 / p}\|x\| \quad(x \in X), \\
\left\|S_{01}\left(\left(z_{i}\right)\right)\right\|_{L^{p}} & =\left\|\sum_{i} r_{i} J_{i}\left(z_{i}\right)\right\|_{L^{p}} \leq \sum_{i} r_{i}\left\|z_{i}\right\|_{F_{i}} \leq M^{1 / q}\left\|\left(z_{i}\right)\right\|_{F} \quad\left(\left(z_{i}\right) \in F\right),
\end{aligned}
$$

and $S_{01} S_{02}=S_{0}$. The rest is clear.

Now suppose $S$ is a $p$-convex regular kernel operator. If $S_{+}^{(1)} S_{+}^{(2)}$ and $S_{-}^{(1)} S_{-}^{(2)}$ are $r$ factorizations of $S_{+}$and $S_{-}$, respectively, through $\ell_{p}$, then noting that $\ell_{p} \oplus_{p} \ell_{p} \cong \ell_{p}$ as Banach lattices, one readily sees that $T_{1}: X \rightarrow \ell_{p} \oplus_{p} \ell_{p}, x \mapsto\left(S_{+}^{(1)} x, S_{-}^{(1)} x\right)$, and $T_{2}: \ell_{p} \oplus_{p} \ell_{p} \rightarrow L^{p}(\mu),\left(\xi_{1}, \xi_{2}\right) \mapsto R S_{+}^{(2)} \xi_{1}-R S_{-}^{(2)} \xi_{2}$, provide the required $r$-factorization of $T$ through $\ell_{p}$.

In the case where $Y$ is $p$-concave and $R: L^{p}(\mu) \rightarrow Y$ is a regular kernel operator, one simply applies the previous part of the argument to the adjoint $R^{\prime}: Y^{\prime} \rightarrow L^{p}(\mu)^{\prime}=L^{q}(\mu)$ to obtain regular maps $R_{1}: \ell_{q} \rightarrow L^{q}(\mu)$ and $R_{2}: Y^{\prime} \rightarrow \ell_{q}$ such that $R^{\prime}=R_{1} R_{2}$ (by [10, Proposition 1.d.4(iii)], $Y$ is $p$-concave $\Longleftrightarrow Y^{\prime}$ is $q$-convex). Since $Y$ is $p$-concave and $p<\infty, Y$ is a KB-space and therefore a band in $Y^{\prime \prime}$. Let $P: Y^{\prime \prime} \rightarrow Y$ be the corresponding band projection. Then $R=\left(P \circ R_{2}^{\prime}\right) \circ R_{1}^{\prime}$, which is an $r$-factorization through $\ell_{p}$.

Remark 3 If $p=\infty$ and $\mu$ and $\nu$ are $\sigma$-finite measures, then an argument similar to (but simpler than) the one used in the first part of the proof of Theorem 1, gives that every kernel operator $T: L^{\infty}(\mu) \rightarrow L^{\infty}(v) r$-factors, or equivalently, factors (see $\left[1\right.$, Theorem 9]) through $\ell_{\infty}$.

As a first consequence of Theorem 1, we now have the following.

Corollary 1 Let $X$ (resp. Y) be a p-convex (resp. p-concave) Banach lattice, with $1<p<\infty$, and let $\mu$ be a $\sigma$-finite measure. Then

$$
\begin{aligned}
\mathcal{I}^{r}\left(X, L^{p}(\mu)\right) & =\Gamma_{\ell_{p}, r}^{o}\left(X, L^{p}(\mu)\right)=\Gamma_{\ell_{p}, r}\left(X, L^{p}(\mu)\right) \\
\left(\operatorname{resp} . \mathcal{I}^{r}\left(L^{p}(\mu), Y\right)\right. & \left.=\Gamma_{\ell_{p}, r}^{o}\left(L^{p}(\mu), Y\right)=\Gamma_{\ell_{p}, r}\left(L^{p}(\mu), Y\right)\right) .
\end{aligned}
$$

In particular, $\mathcal{I}^{r}\left(L^{p}(\mu)\right)=\Gamma_{\ell_{p}, r}^{o}\left(L^{p}(\mu)\right)=\Gamma_{\ell_{p}, r}\left(L^{p}(\mu)\right)(1<p<\infty)$.

Proof Suppose first $X$ is $p$-convex. By Theorem $1, \mathcal{I}^{r}\left(X, \ell_{p}\right) \subseteq \Gamma_{\ell_{p}, r}\left(X, L^{p}(\mu)\right)$, and since $\mathcal{I}^{r}\left(X, \ell_{p}\right)$ is a band, it will suffice to show that $\Gamma_{\ell_{p}, r}\left(X, L^{p}(\mu)\right) \subseteq \mathcal{I}^{r}\left(X, \ell_{p}\right)$, for then one has that $\Gamma_{\ell_{p}, r}^{\mathrm{o}}\left(X, L^{p}(\mu)\right) \subseteq \mathcal{F}\left(X, L^{p}(\mu)\right)^{d d}$, and clearly, $\Gamma_{\ell_{p}, r}\left(X, L^{p}(\mu)\right) \subseteq$ 
$\Gamma_{\ell_{p}, r}^{\mathrm{o}}\left(X, L^{p}(\mu)\right)$. In turn, by Remark 1 , we only need to check that if an operator $T: X \rightarrow L^{p}(\mu) r$-factors through $\ell_{p}$, then $T \in \mathcal{I}^{r}\left(X, \ell_{p}\right)$. But $\ell_{p}$ is order continuous and atomic, so $\mathcal{I}^{r}\left(X, \ell_{p}\right)=\mathcal{L}^{r}\left(X, \ell_{p}\right)$, and therefore $\mathcal{L}^{r}\left(\ell_{p}, L^{p}(\mu)\right) \circ \mathcal{L}^{r}\left(X, \ell_{p}\right)=$ $\mathcal{L}^{r}\left(\ell_{p}, L^{p}(\mu)\right) \circ \mathcal{I}^{r}\left(X, \ell_{p}\right) \subseteq \mathcal{I}^{r}\left(X, L^{p}(\mu)\right)$.

The proof that $\mathcal{I}^{r}\left(L^{p}(\mu), Y\right)=\Gamma_{\ell_{p}, r}^{\mathrm{o}}\left(L^{p}(\mu), Y\right)=\Gamma_{\ell_{p}, r}\left(L^{p}(\mu), Y\right)$ if $Y$ is $p$ concave, is completely analogous. The rest is clear.

Remark 4 Since $\ell_{1}$ is order continuous and, as noted at the beginning of this section, if $\mu$ and $v$ are finite measures, every kernel operator $T: L^{1}(\mu) \rightarrow L^{1}(\nu) r$-factors (or equivalently, factors) through $\ell_{1}$ (see [1, Theorem 9]), the last part of Corollary 1 holds true also for $p=1$ when $\mu$ is finite.

It should be clear now, from Corollary 1 , that in order to show $\mathcal{A}^{r}\left(L^{p}(\mu)\right)$ is the only non-zero proper closed ideal of $\mathcal{I}^{r}\left(L^{p}(\mu)\right.$ ) (with $\mu$ as in Corollary 1) it would suffice to show that $\mathrm{id}_{\ell_{p}} r$-factors through any linear map in $\mathcal{I}^{r}\left(L^{p}(\mu)\right) \backslash \mathcal{A}^{r}\left(L^{p}(\mu)\right)$. We shall prove this below for any $1<p<\infty$. In fact, for $p \neq 2$, slightly more can be said.

Before stating our next result, we recall a few things. First, a sequence $\left(x_{i}\right) \subset X$ is said to be complemented by a projection, or just complemented, if $\left[x_{i}\right]$ is the image of a bounded projection on $X$. Second, an order continuous Banach lattice $X$ is said to have the subsequence splitting property (SSP in short) if for every bounded sequence $\left(x_{n}\right) \subset X$ there is an increasing sequence $\left(n_{i}\right) \subset \mathbb{N}$, an equi-integrable sequence $\left(f_{i}\right) \subset X$ (i.e., a sequence such that for some $u \in X_{+}, \lim _{t \rightarrow \infty} \sup _{k} \|\left|f_{k}\right|-\left|f_{k}\right| \wedge$ $t u \|=0)$ and a disjoint sequence $\left(g_{i}\right) \subset X$ such that $\left|f_{i}\right| \wedge\left|g_{i}\right|=0$ and $x_{n_{i}}=f_{i}+g_{i}$ $(i \in \mathbb{N}$ ). (This is the same as [19, Definition 2.1], except for the fact that it has not been restricted to order continuous Banach lattices with weak order units as in [19]). In view of [10, Proposition 1.a.9] and the results of [19], it is clear that any $q$-concave Banach lattice, with $q<\infty$, has the SSP. Third, the lower index, $s(X)$, of a Banach lattice $X$ is defined as $s(X):=\sup \{p \in[1, \infty]: X$ satisfies an upper $p$-estimate $\}$, where $X$ is said to satisfy an upper $p$-estimate if for some constant $C$ and every finite disjoint sequence $x_{1}, \ldots, x_{n} \in X,\left\|\sum_{i=1}^{n} x_{i}\right\| \leq C\left(\sum_{i=1}^{n}\left\|x_{i}\right\|^{p}\right)^{1 / p}$. Lastly, recall $T \in \mathcal{L}^{r}(X, Y)$ is AM-compact if it maps order bounded sets to precompact ones. It is easy to see (and well known) that an AM-compact operator must also map semicompact sets to precompact ones, where by a semicompact set we mean a subset $A \subset X$ with the property that for every $\varepsilon>0$ there exists a $u \in X_{+}$such that $\left\|(|\xi|-u)_{+}\right\|<\varepsilon(\xi \in A)$. If $Y$ is order continuous, then the space $\mathcal{A M}(X, Y)$ of all AM-compact operators from $X$ to $Y$ is a band in $\mathcal{L}^{r}(X, Y)$ [7, Theorem 4.7], and hence, $\mathcal{I}^{r}(X, Y) \subseteq \mathcal{A M}(X, Y)$.

Proposition 1 Let $X$ and $Y$ be Banach lattices. Suppose there are positive disjoint normalized sequences $\left(\xi_{i}\right) \subset X$ and $\left(\eta_{i}\right) \subset Y$ satisfying:

- For every positive disjoint normalized sequence $\mathbf{x}=\left(x_{i}\right) \subset X$ there is a constant $C=C(\mathbf{x})$ such that $\left\|\sum_{i} \alpha_{i} x_{i}\right\| \leq C\left\|\sum_{i} \alpha_{i} \xi_{i}\right\|\left(\left(\alpha_{i}\right) \in c_{00}\right) ;$

- For every positive disjoint normalized sequence $\mathbf{y}=\left(y_{i}\right) \subset Y$ there is a constant $C=C(\mathbf{y})$ such that $\left\|\sum_{i} \alpha_{i} \eta_{i}\right\| \leq C\left\|\sum_{i} \alpha_{i} y_{i}\right\|\left(\left(\alpha_{i}\right) \in c_{00}\right)$;

- There is a constant $M$ such that $\left\|\sum_{i} \alpha_{i} \eta_{i}\right\| \leq M\left\|\sum_{i} \alpha_{i} \xi_{i}\right\|\left(\left(\alpha_{i}\right) \in c_{00}\right)$. 
Furthermore, suppose $X$ is order continuous, has the $S S P, s(X)>2$ and every positive disjoint normalized sequence in $Y$ contains a subsequence complemented by a regular projection. Then $K:\left[\xi_{i}\right] \rightarrow\left[\eta_{i}\right], \sum_{i} \alpha_{i} \xi_{i} \mapsto \sum_{i} \alpha_{i} \eta_{i}, r$-factors through every positive noncompact AM-compact operator $T: X \rightarrow Y$.

Proof Let $X$ and $Y$ be Banach lattices as in the hypotheses and let $T \in \mathcal{L}^{r}(X, Y)_{+}$be a noncompact AM-compact operator. Since $T$ is noncompact, there must be a sequence $\left(x_{k}\right)$ in $X_{+}$such that $\left(T x_{k}\right)$ has no Cauchy subsequences, and since $X$ has the SSP, $\left(x_{k}\right)$ has a subsequence $\left(x_{k_{n}}\right)$ such that $\left(x_{k_{n}}\right)=\left(f_{n}\right)+\left(g_{n}\right)$, with $\left(f_{n}\right) \subset X$ equi-integrable, $\left(g_{n}\right) \subset X$ disjoint and $f_{n} \wedge g_{n}=0(n \in \mathbb{N})$. Next, as $T$ is AM-compact, $\left\{T f_{n}: n \in \mathbb{N}\right\}$ must be precompact (recall $T$ must map semicompact sets to precompact ones), and so, $\left(g_{n}\right)$ must contain a subsequence $\left(g_{n_{i}}\right)$ such that $\inf _{i}\left\|T g_{n_{i}}\right\|=: \delta>0$.

Set $y_{i}:=T g_{n_{i}}(i \in \mathbb{N})$. By [10, Proposition 1.c.10], either $\left(y_{i}\right)$ contains an asymptotically disjoint subsequence (i.e., a subsequence $\left(y_{i_{k}}\right)$ such that $\lim _{k}\left\|y_{i_{k}}-\phi_{k}\right\|=0$ for some disjoint sequence $\left(\phi_{k}\right) \subset Y$ ) or there is a constant $C$ such that

$$
\left(\sum_{i=1}^{N}\left|\alpha_{i}\right|^{2}\right)^{\frac{1}{2}} \leq C 2^{-N} \sum_{\varepsilon_{i}= \pm 1}\left\|\sum_{i=1}^{N} \varepsilon_{i} \alpha_{i} y_{i}\right\| \leq C M\|T\|\left\|\sum_{i=1}^{N} \alpha_{i} g_{n_{i}}\right\|,
$$

for every set of scalars $\alpha_{1}, \ldots, \alpha_{N}$ and $N \in \mathbb{N}$, where $M$ stands for the unconditionality constant of $\left(g_{n_{i}}\right)$. But $s(X)>2$, so $\left(y_{i}\right)$ must contain an asymptotically disjoint subsequence $\left(y_{i_{k}}\right)$. In view of our hypotheses on $Y$, by further passing to a subsequence if necessary, we can assume there is a positive disjoint sequence $\left(\psi_{k}\right) \subset Y$, complemented by a regular projection $Q$ and such that $\sum_{k}\left\|y_{i_{k}}-\psi_{k}\right\|<\delta / 2$.

Since $\left(\psi_{k}\right)$ is a 1-unconditional basis for $\left[\psi_{k}\right]$, each biorthogonal functional $\psi_{k}^{*}$ has norm $\leq\left\|\psi_{k}\right\|^{-1}<2 / \delta\left(\right.$ for $\inf _{i}\left\|y_{i}\right\|=\delta$ and $\left.\left\|y_{i_{k}}-\psi_{k}\right\|<\delta / 2(k \in \mathbb{N})\right)$, and by [6, Chapter V, Theorem 9], $\left(y_{i_{k}}\right)$ is a basic sequence equivalent to $\left(\psi_{k}\right)$, and hence a basis for $\left[y_{i_{k}}\right]$. For each $k \in \mathbb{N}$, let $z_{k}^{*}$ be any norm-preserving extension to $Y$, of the $k$-th biorthogonal functional on $\left[y_{i_{k}}\right]$ corresponding to the basis $\left(y_{i_{k}}\right)$. Note $\left(z_{k}^{*}\right)$ is norm-bounded, for if $L:\left[y_{i_{k}}\right] \rightarrow\left[\psi_{k}\right], \sum_{k} \alpha_{k} y_{i_{k}} \mapsto \sum_{k} \alpha_{k} \psi_{k}$, then $y_{i_{k}}^{*}=\psi_{k}^{*} \circ L$ $(k \in \mathbb{N})$.

To finish, let $R_{1}:\left[\xi_{k}\right] \rightarrow X, \sum_{k} \alpha_{k} \xi_{k} \mapsto \sum_{k} \alpha_{k} g_{n_{i_{k}}}$, let $S_{1}: Y \rightarrow Y, y \mapsto$ $y+\sum_{k} z_{k}^{*}(y)\left(\psi_{k}-y_{i_{k}}\right)$ and let $S_{2}:\left[\psi_{k}\right] \rightarrow\left[\eta_{k}\right], \sum_{k} \alpha_{k} \psi_{k} \mapsto \sum_{k} \alpha_{k} \eta_{k}$. All the maps just defined are readily seen to be well-defined and regular (in the case of $S_{1}$ simply note that it is a nuclear perturbation of $\mathrm{id}_{Y}$ ) and one easily checks that $S_{2} Q S_{1} T R_{1}=K$.

Corollary 2 Let $p, q$ be such that either $1<p \leq q<2$ or $2<p \leq q<\infty$, let $\iota_{p q}: \ell_{p} \rightarrow \ell_{q}$ be the natural embedding (of course, $\iota_{p p}$ is just $\mathrm{id}_{\ell_{p}}$ ), and let $\mu$ and $v$ be $\sigma$-finite measures. If $L^{p}(\mu)$ and $L^{q}(v)$ are infinite dimensional, then $\mathcal{I}:=$ $\Gamma_{l p q, r}^{o}\left(L^{p}(\mu), L^{q}(v)\right)$ is the smallest order ideal of $\mathcal{I}^{r}\left(L^{p}(\mu), L^{q}(v)\right)$, not contained in $\mathcal{A}^{r}\left(L^{p}(\mu), L^{q}(v)\right)$ and with the property that $\mathcal{L}^{r}\left(L^{q}(v)\right) \circ \mathcal{I} \circ \mathcal{L}^{r}\left(L^{p}(\mu)\right) \subseteq \mathcal{I}$.

Proof Suppose first $2<p \leq q<\infty$. Let $\left(\xi_{i}\right)$ and $\left(\eta_{i}\right)$ be positive disjoint normalized sequences in $L^{p}(\mu)$ and $L^{q}(\nu)$, respectively. Let $\phi:\left[\xi_{i}\right] \rightarrow \ell_{p}$, $\sum_{i} \alpha_{i} \xi_{i} \mapsto\left(\alpha_{i}\right)$, and $\psi: \ell_{q} \rightarrow\left[\eta_{i}\right],\left(\beta_{i}\right) \mapsto \sum_{i} \beta_{i} \eta_{i}$. By Proposition 1 , if $T \in$ 
$\mathcal{L}^{r}\left(L^{p}(\mu), L^{q}(v)\right)_{+}$is noncompact AM-compact then there are $V \in \mathcal{L}^{r}\left(L^{q}(\nu),\left[\eta_{i}\right]\right)$ and $W \in \mathcal{L}^{r}\left(\left[\xi_{i}\right], L^{p}(\mu)\right)$ so that $V T W=\psi \iota_{p q} \phi$. In turn, $\iota_{p q}=\psi^{-1} V T W \phi^{-1}$, and therefore, $\Gamma_{l p q, r}\left(L^{p}(\mu), L^{q}(\nu)\right) \subseteq \Gamma_{T, r}\left(L^{p}(\mu), L^{q}(\nu)\right)$.

Now let $T \in \mathcal{I}^{r}\left(L^{p}(\mu), L^{q}(v)\right) \backslash \mathcal{A}^{r}\left(L^{p}(\mu), L^{q}(v)\right)$ arbitrary. Then $|T|$ is a noncompact AM-compact operator. The smallest order ideal $\mathcal{J}$ of $\mathcal{L}^{r}\left(L^{p}(\mu), L^{q}(\nu)\right)$ that contains $T$ and satisfies $\mathcal{L}^{r}\left(L^{q}(v)\right) \circ \mathcal{J} \circ \mathcal{L}^{r}\left(L^{p}(\mu)\right) \subseteq \mathcal{J}$ is clearly $\Gamma_{|T|, r}^{o}\left(L^{p}(\mu), L^{q}(\nu)\right)$, and by the result from the previous paragraph, $\Gamma_{l_{p q}, r}\left(L^{p}(\mu), L^{q}(\nu)\right) \subseteq \Gamma_{|T|, r}\left(L^{p}(\mu), L^{q}(\nu)\right)$. From this, one readily obtains that $\mathcal{I} \subseteq \Gamma_{|T|, r}^{o}\left(L^{p}(\mu), L^{q}(\nu)\right)$. Since the last holds for any $T \in \mathcal{I}^{r}\left(L^{p}(\mu), L^{q}(\nu)\right) \backslash$ $\mathcal{A}^{r}\left(L^{p}(\mu), L^{q}(\nu)\right)$ and $\mathcal{I}$ is an order ideal not contained in $\mathcal{A}^{r}\left(L^{p}(\mu), L^{q}(v)\right)$ and such that $\mathcal{L}^{r}\left(L^{q}(v)\right) \circ \mathcal{I} \circ \mathcal{L}^{r}\left(L^{p}(\mu)\right) \subseteq \mathcal{I}$, the desired conclusion follows for $2<p \leq q<\infty$.

Second, suppose $1<p \leq q<2$ and let $T \in \mathcal{L}^{r}\left(L^{p}(\mu), L^{q}(\nu)\right)_{+}$be a noncompact AM-compact operator. Then $2<q^{\prime} \leq p^{\prime}<\infty$ and $T^{\prime} \in \mathcal{L}^{r}\left(L^{q^{\prime}}(v), L^{p^{\prime}}(\mu)\right)_{+}$is also noncompact AM-compact (see [22, Theorem 125.6]), where $p^{\prime}$ and $q^{\prime}$ stand for the conjugates of $p$ and $q$, respectively. As in the previous case, $\imath_{q^{\prime}} p^{\prime}=R T^{\prime} S$ for some $R \in \mathcal{L}^{r}\left(L^{p^{\prime}}(\mu), \ell_{p^{\prime}}\right)$ and $S \in \mathcal{L}^{r}\left(\ell_{q^{\prime}}, L^{q^{\prime}}(v)\right)$. Taking adjoints on the last identity, one obtains that $\iota_{p q}=S^{\prime} T R^{\prime}$, and in turn, that $\Gamma_{l_{p q}, r}\left(L^{p}(\mu), L^{q}(\nu)\right) \subseteq$ $\Gamma_{T, r}\left(L^{p}(\mu), L^{q}(\nu)\right)$. From this, arguing exactly as in the previous paragraph, one obtains the desired conclusion for $1<p \leq q<2$.

Unfortunately, except for the case where $p=2=q$, which we discuss next, we are unable to shed any more light into what happens when $1<p \leq 2 \leq q<\infty$. Our next proposition strongly suggests the need for a different approach in these cases.

Proposition 2 Let $L^{2}(\mu)$ be infinite-dimensional, with $\mu \sigma$-finite, let $\left(\chi_{i}\right)$ be a disjoint sequence of non-zero characteristic functions and let $P$ be the average projection defined by $P(f):=\sum_{i}\left\|\chi_{i}\right\|^{-2}\left(\int f \chi_{i} d \mu\right) \chi_{i}\left(f \in L^{2}(\mu)\right)$. If $T \in \mathcal{L}^{r}\left(L^{2}(\mu)\right)$ is a positive noncompact kernel operator then $\Gamma_{T, r}^{o}\left(L^{2}(\mu)\right) \ni P$.

Proof Let $X:=L^{2}(\mu)$ and let $T \in \mathcal{L}^{r}(X)_{+}$be a noncompact kernel operator. Let $\delta:=\inf \left\{\|T-S\|: S \in \mathcal{A}^{r}(X) \cap[0, T]\right\}$. Note $\delta>0$ since otherwise $T$ would be compact. Let $\mathfrak{M} \subset X$ be a complete orthonormal system for $X$, and for every $E \subset \mathfrak{M}$, let $P_{E}$ be the natural projection onto $\operatorname{sp}(E)$ with respect to $\mathfrak{M}$.

Given $\varepsilon>0$, we construct positive sequences $\left(\xi_{i}\right) \subset X$ and $\left(\phi_{i}\right) \subset X^{\prime}$, and an increasing sequence of finite subsets of $\mathfrak{M}$, as follows. First, set $T_{1}:=T$ and choose $\xi_{1} \in X_{[1]} \cap X_{+}$and $\phi_{1} \in X_{[1]}^{\prime} \cap X_{+}^{\prime}$ so that $\phi_{1}\left(T_{1} \xi_{1}\right) \geq\left\|T_{1}\right\|-\varepsilon / 2$. Next, choose $F_{1} \subset \mathfrak{M}$ finite so that

$$
\max \left\{\left\|\xi_{1}-P_{F_{1}} \xi_{1}\right\|,\left\|\phi_{1}-\phi_{1} P_{F_{1}}\right\|\right\} \leq \frac{\varepsilon}{2\left\|T_{1}\right\|}
$$

and then choose $S_{1} \in \mathcal{A}^{r}(X) \cap\left[0, T_{1}\right]$ so that

$$
\max \left\{\left\|P_{F_{1}}\left(T_{1}-S_{1}\right)\right\|,\left\|\left(T_{1}-S_{1}\right) P_{F_{1}}\right\|\right\} \leq \frac{\varepsilon}{2} .
$$


(To see the latter is possible, recall $\mathcal{A}^{r}(X)$ is an order ideal whenever $X$ and $X^{\prime}$ are both order continuous, and so, $T=\sup \left([0, T] \cap \mathcal{A}^{r}(X)\right)$ [13, Proposition 1.2.6].) In turn, $T x=\sup \left\{S x: S \in[0, T] \cap \mathcal{A}^{r}(X)\right\}\left(x \in X_{+}\right)$, and since $X$ is ordercontinuous, $\lim _{S \in \mathfrak{S}}\|T x-S x\|=0\left(x \in X_{+}\right)$, where we have written $\mathfrak{S}$ for the upwards directed set $[0, T] \cap \mathcal{A}^{r}(X)$. If $\Delta$ is an $\bar{\varepsilon}$-net for $P_{F_{1}}\left(X_{[1]}\right)$, then, by the previous discussion, there is an $S \in \mathcal{A}^{r}(X)$ so that $\|(T-S)(x)\|<\bar{\varepsilon}(x \in \Delta)$, and hence, so that $\left\|(T-S) P_{F_{1}}\right\|<(1+\|T\|) \bar{\varepsilon}$. As for the other inequality, note $T^{\prime}$ is a kernel operator ([22, Theorem 97.3]), so, by the previous part, there is for every $\bar{\varepsilon}>0$ an operator $R \in \mathcal{A}^{r}\left(X^{\prime}\right)$ so that $\left\|\left(T^{\prime}-R\right) P_{F_{1}}^{\prime}\right\|<\bar{\varepsilon}$. Then note that $\left\|P_{F_{1}}\left(T-R^{\prime}\right)\right\|=\left\|\left(T^{\prime}-R\right) P_{F_{1}}^{\prime}\right\|$ and $R^{\prime} \in \mathcal{A}^{r}(X)$.) Set $T_{2}:=T_{1}-S_{1}$. In general, if $T_{i} \in \mathcal{L}^{r}(X)_{+}$has been defined and $F_{i-1} \subset \mathfrak{M}$ has been chosen, choose $\xi_{i} \in X_{[1]} \cap X_{+}$ and $\phi_{i} \in X_{[1]}^{\prime} \cap X_{+}^{\prime}$ so that $\phi_{i}\left(T_{i} \xi_{i}\right) \geq\left\|T_{i}\right\|-\varepsilon / 2^{i}$. Then choose $F_{i} \supset F_{i-1}$ finite so that

$$
\max \left\{\left\|\xi_{j}-P_{F_{i}} \xi_{j}\right\|,\left\|\phi_{j}-\phi_{j} P_{F_{i}}\right\|\right\} \leq \frac{\varepsilon}{2^{i}\left\|T_{i}\right\|} \quad(1 \leq j \leq i)
$$

choose $S_{i} \in \mathcal{A}^{r}(X) \cap\left[0, T_{i}\right]$ so that

$$
\max \left\{\left\|P_{F_{i}}\left(T_{i}-S_{i}\right)\right\|,\left\|\left(T_{i}-S_{i}\right) P_{F_{i}}\right\|\right\} \leq \frac{\varepsilon}{2^{i}},
$$

and set $T_{i+1}:=T_{i}-S_{i}$. Further, set $P_{F_{0}}=T_{0}=S_{0}=0$, and to simplify notations set $Q_{i}:=P_{F_{i}}-P_{F_{i-1}}(i \in \mathbb{N})$.

Let $F:=\bigcup_{i} F_{i}$. Then, for every $i \in \mathbb{N}$,

$$
\begin{aligned}
\left\|T_{i}\right\|^{2}\left(1-\left\|\left(P_{F}-Q_{i}\right) \xi_{i}\right\|^{2}\right) & \geq\left\|T_{i}\right\|^{2}\left\|Q_{i} \xi_{i}\right\|^{2} \geq\left\|T_{i} Q_{i} \xi_{i}\right\|^{2} \\
& \geq\left(\left\|T_{i} P_{F_{i}} \xi_{i}\right\|-\left\|\left(T_{i-1}-S_{i-1}\right) P_{F_{i-1}}\right\|\right)^{2} \\
& \geq\left(\left\|T_{i}\right\|-\frac{\varepsilon}{2^{i-2}}\right)^{2}
\end{aligned}
$$

and so,

$$
\left\|\left(P_{F}-Q_{i}\right) \xi_{i}\right\|^{2} \leq 1-\left(1-\frac{\varepsilon}{2^{i-2}\left\|T_{i}\right\|}\right)^{2} \leq \frac{\varepsilon}{2^{i-3} \delta}
$$

In turn, $\sum_{i}\left\|\xi_{i}-Q_{i} \xi_{i}\right\|<8 \sqrt{\varepsilon / \delta}$ (note that $P_{F} \xi_{i}=\xi_{i}(i \in \mathbb{N}$ )). On the other hand, it follows from our construction of $\left(\xi_{i}\right)$ that $\|T\|\left\|\xi_{i}\right\| \geq\left\|T_{i}\right\|-\varepsilon / 2^{i} \geq \delta / 2(i \in \mathbb{N})$, and hence, that $\left\|Q_{i} \xi_{i}\right\| \geq \delta /(2\|T\|)-2 \sqrt{\varepsilon / \delta}(i \in \mathbb{N})$.

Write $F$ as a sequence $\left(x_{i}\right)$ in such a way that if $\left|F_{k-1}\right|<i \leq\left|F_{k}\right|$ then $x_{i} \in$ $F_{k} \backslash F_{k-1}(k \in \mathbb{N})$, with $F_{0}:=\emptyset$. Let us suppose $\delta /(2\|T\|)>2 \sqrt{\varepsilon / \delta}$, so $Q_{i} \xi_{i} \neq 0$ $(i \in \mathbb{N})$. In this situation, it is clear that $\left(Q_{i} \xi_{i}\right)$ is a block basic sequence with respect to $\left(x_{i}\right)$, whose unconditionality constant equals 1 . If $f_{i}$ denotes the $i$-th biorthogonal functional on $\left[Q_{i} \xi_{i}\right]$, corresponding to the basis $\left(Q_{i} \xi_{i}\right)$, then $\left\|f_{i}\right\| \leq 1 /\left\|Q_{i} \xi_{i}\right\|(i \in \mathbb{N})$ and from our previous estimates we obtain that 


$$
\sum_{i}\left\|f_{i}\right\|\left\|\xi_{i}-Q_{i} \xi_{i}\right\| \leq \frac{16\|T\| \sqrt{\varepsilon}}{\delta^{\frac{3}{2}}-4\|T\| \sqrt{\varepsilon}}
$$

So provided $\varepsilon$ is small enough, $\sum_{i}\left\|f_{i}\right\|\left\|\xi_{i}-Q_{i} \xi_{i}\right\|<1$ and $\left(\xi_{i}\right)$ is a basic sequence in $X$, equivalent to $\left(Q_{i} \xi_{i}\right)$ (see [6, Chapter V, Theorem 9]). Analogously, one shows $\left(\phi_{i}\right)$ is equivalent to $\left(Q_{i}^{\prime} \phi_{i}\right)$ for every $\varepsilon>0$ sufficiently small.

Suppose $\varepsilon$ has been chosen so that $\left(\xi_{i}\right)$ and $\left(\phi_{i}\right)$ are basic sequences equivalent to $\left(Q_{i} \xi_{i}\right)$ and $\left(Q_{i}^{\prime} \phi_{i}\right)$, respectively. Define $U: X \rightarrow X$ by $U f:=$ $\sum_{i}\left\|\chi_{i}\right\|^{-1}\left(\int f \chi_{i} d \mu\right) \xi_{i}(f \in X)$ and $V: X \rightarrow X$ by $V f:=\sum_{i}\left\|\chi_{i}\right\|^{-1} \phi_{i}(f) \chi_{i}$ $(f \in X)$. It is easy to see that both $\left(Q_{i} \xi_{i}\right)$ and $\left(Q_{i}^{\prime} \phi_{i}\right)$ are equivalent to the unit vector basis of $\ell_{2}$, and hence, to $\left(\left\|\chi_{i}\right\|^{-1} \chi_{i}\right)$. In turn, $\left(\xi_{i}\right)$ and $\left(\phi_{i}\right)$ are equivalent to ( $\left\|\chi_{i}\right\|^{-1} \chi_{i}$ ), and one readily sees $U$ and $V$ are well-defined. Clearly, $U$ and $V$ are positive (hence, regular). To finish the proof, simply note that $\phi_{i}\left(T \xi_{i}\right) \geq \phi_{i}\left(T_{i} \xi_{i}\right) \geq$ $\left\|T_{i}\right\|-\varepsilon / 2^{i}>\delta / 2(i \in \mathbb{N})$, so

$$
V T U f \geq \sum_{i} \phi_{i}\left(T \xi_{i}\right)\left\|\chi_{i}\right\|^{-2}\left(\int f \chi_{i} d \mu\right) \chi_{i} \geq \frac{\delta}{2} P f \quad\left(f \in X_{+}\right) .
$$

The rest is clear.

The bimonotonicity of the basic sequence $\left(x_{i}\right)$, in the proof of Proposition 2, although (admittedly) not entirely apparent, played a key role. For $p \neq 2$, no basis for a separable non-atomic $L^{p}$-space can be bimonotone (recall the Haar system in $L^{p}[0,1]$ is precisely reproducible [10, Theorem 2.c.8]; and is bimonotone only if $p=2$ [see [14] for a much stronger result)]. This appears to be the main obstacle preventing the above argument from working for non-atomic $L^{p}$-spaces when $p \neq 2$.

We can now prove the following result, announced in the abstract:

Corollary 3 If $\mu$ is $\sigma$-finite then $\mathcal{A}^{r}\left(L^{p}(\mu)\right)$ is the only non-zero proper closed ideal (i.e., order and algebra ideal) of $\mathcal{I}^{r}\left(L^{p}(\mu)\right)$ for every $1<p<\infty$.

Proof First, by Corollary 1, for every $1<p<\infty, \mathcal{I}^{r}\left(L^{p}(\mu)\right)=\Gamma_{\ell_{p}, r}^{o}\left(L^{p}(\mu)\right)$. If either $1<p<2$ or $2<p<\infty$, then, by Corollary $2, \Gamma_{\ell_{p}, r}^{o}\left(L^{p}(\mu)\right)$ is the smallest ideal of $\mathcal{I}^{r}\left(L^{p}(\mu)\right)$ not contained in $\mathcal{A}^{r}\left(L^{p}(\mu)\right)$, and the desired conclusion follows. If on the other hand, $p=2$, then, by Proposition 2 , the order and algebra ideal of $\mathcal{L}^{r}\left(L^{2}(\mu)\right)$ generated by an operator $T \in \mathcal{I}^{r}\left(L^{2}(\mu)\right) \backslash \mathcal{A}^{r}\left(L^{2}(\mu)\right)$ must contain a positive projection $P$ whose range is the span of a normalized disjoint sequence in $L^{2}(\mu)_{+}$, and therefore, isometrically Riesz isomorphic to $\ell_{2}$. It follows readily from this that any operator in $\mathcal{L}^{r}\left(L^{2}(\mu)\right), r$-factoring through $\ell_{2}, r$-factors through $P$, and hence, that $\Gamma_{\ell_{2}, r}^{o}\left(L^{2}(\mu)\right) \subseteq \Gamma_{T, r}^{o}\left(L^{2}(\mu)\right)$. The rest is clear.

The result of Corollary 3 fails for non-atomic $L^{1}$-spaces since, in this case, the approximable regular operators no longer form an order ideal but just a Banach lattice. However, it is still true that if $X$ is an $L^{1}(\mu)$-space, with $\mu$-finite, then there is only one closed non-trivial proper (order and algebra) ideal in the band of kernel operators. We suspect this is probably known, although we do not have a reference for it. Clearly, 
it does not follow from our previous results, so, for completeness, we provide next a sketched proof of it.

To fix ideas, let $X$ be an $L^{1}(\mu)$-space with $\mu$ finite. Recall first that the algebra ideal of weakly compact operators on $X, \mathcal{W}(X)$, is an order ideal (see [2, Corollary 5.36]) and satisfies $\mathcal{W}(X) \subsetneq \mathcal{I}^{r}(X)$ (see for instance [13, Theorem 3.5.16]). It turns out that

$\mathcal{W}(X)$ is the only closed non-trivial proper ideal of $\mathcal{I}^{r}(X)$.

Proof We show first that $\mathcal{W}(X)$ is the smallest norm-closed order ideal of $\mathcal{I}^{r}(X)$ that contains $\mathcal{F}(X)$. Clearly, it will suffice to show that every $T \in \mathcal{W}(X)_{+}$belongs to the closed order ideal generated by $\mathcal{F}(X)$. So let $T \in \mathcal{W}(X)_{+}$and $\varepsilon>0$ arbitrary. Now, as $T$ is weakly compact, by [9, Theorem III.2.12], there is $k_{T} \in L^{\infty}(\mu, X)$ such that $T f=\int_{\Omega} k_{T} f d \mu(f \in X)$ and $\left\{k_{T}(t): t \in \Omega\right\}$ is relatively weakly compact in $X$ (where $\Omega$ stands for the underlying set of the measure space). Furthermore, since relatively weakly compact subsets of $X$ are semicompact (see [13, Theorem 2.5.4]), there exists $u \in X_{+}$such that $\left\{k_{T}(t): t \in \Omega\right\} \subseteq[-u, u]+\varepsilon X_{[1]}$. Define $S f:=$ $\left(\int_{\Omega} f d \mu\right) u(f \in X)$. Then $S \in \mathcal{F}(X)$ and for every $f \in X_{+}$,

$$
\begin{aligned}
\left\|(T-S)_{+}(f)\right\|_{1} & \leq\left\|\int_{\Omega}\left(k_{T}(t)-u\right)_{+} f(t) d \mu(t)\right\|_{1} \\
& \leq \int_{\Omega}\left\|\left(k_{T}(t)-u\right)_{+}\right\|_{1} f(t) d \mu(t) \leq \varepsilon\|f\|_{1}
\end{aligned}
$$

(the first inequality follows easily from the fact that $|(T-S)| f=\sup _{|\xi| \leq f}|(T-S) \xi| \leq$ $\left.\int\left|k_{T}(t)-u\right| f(t) d \mu\right)$. One concludes readily from this that $\left\|(T-S)_{+}\right\| \leq \varepsilon$, and since $T=T \wedge S+(T-S)_{+}$and $\varepsilon>0$ was arbitrary, the desired conclusion follows.

To see $\mathcal{W}(X)$ is also the only closed ideal of $\mathcal{I}^{r}(X)$, let $T \in \mathcal{I}^{r}(X) \backslash \mathcal{W}(X)$ be arbitrary. Since $T\left(X_{[1]}\right)$ is not relatively weakly compact, it must contain a sequence $\left(y_{i}\right)$ equivalent to the unit vector basis of $\ell_{1}$ (see [21, III.C.12]). Let $Y$ be a separable closed Riesz subspace of $X$ containing $\left[y_{i}\right]$. The space $Y$ must be isomorphic, as a Banach space, to either $\ell_{1}$ or $L^{1}[0,1]$, and so, $\left[y_{i}\right]$ must contain a sequence $\left(z_{i}\right)$ equivalent to the unit vector basis of $\ell_{1}$ and such that $\left[z_{i}\right]$ is complemented in $Y$ (see for instance [5, Propositions IV.1.2 \& VI.2.8]). In turn, as $Y$ is complemented in $X$, so is $\left[z_{i}\right]$. Choose a bounded sequence $\left(x_{i}\right) \subset X$ such that $T x_{i}=z_{i}(i \in \mathbb{N})$ (that such a sequence exists, follows on noting that if $\left(\xi_{i}\right) \subset X_{[1]}$ is such $y_{i}=T \xi_{i}(i \in \mathbb{N})$, then $\left(\xi_{i}\right)$ is equivalent to $\left(y_{i}\right)$ and the restriction of $T$ to $\left[\xi_{i}\right]$ is an isomorphism onto $\left.\left[y_{i}\right]\right)$. Let $P: X \rightarrow\left[z_{i}\right]$ be a bounded projection, let $\left(e_{i}\right)$ stand for the unit vector basis of $\ell_{1}$, let $R: \ell_{1} \rightarrow X, \sum_{i} \alpha_{i} e_{i} \mapsto \sum_{i} \alpha_{i} x_{i}$, and let $S:\left[z_{i}\right] \rightarrow \ell_{1}, \sum_{i} \alpha_{i} z_{i} \mapsto \sum_{i} \alpha_{i} e_{i}$. Then $S P T R$ is a factorization of $\operatorname{id}_{\ell_{1}}$ through $T$. Since every integral operator on $L^{1}(\mu)$ must factor through $\ell_{1}$ (see Remark 4), the algebra ideal generated by $T$ must be the whole of $\mathcal{I}^{r}(X)$.

Recall an operator $T \in \mathcal{L}^{r}(X)$ is said to be semicompact if $T\left(X_{[1]}\right)$ is semicompact. The linear $\operatorname{span} \mathcal{S} \mathcal{K}^{r}(X)$ of the positive semicompact operators on a Dedekind complete Banach lattice $X$ is always a norm-closed ideal of $\mathcal{L}^{r}(X)$ (this is well-known and easy to prove). Now let $X$ be an $L^{p}(\mu)$-space for some $1 \leq p<\infty$. If $p=1$ then $\mathcal{S K}^{r}(X)=\mathcal{W}(X)$ (see [13, Theorem 2.5.4] and [2, Theorem 5.35]), while if 
$1<p<\infty$ then $\mathcal{I}^{r}(X) \cap \mathcal{S} \mathcal{K}^{r}(X)=\mathcal{A}^{r}(X)$ [17, Theorem 3.3]. One can thus restate the result of Corollary 3 for finite $\mu$ and the above result, in a unified way, as follows:

Corollary 4 For every $1 \leq p<\infty$ and $\mu$ finite, $\mathcal{I}^{r}\left(L^{p}(\mu)\right) \cap \mathcal{S K}^{r}\left(L^{p}(\mu)\right)$ is the only closed non-trivial proper ideal of $\mathcal{I}^{r}\left(L^{p}(\mu)\right)$.

\section{$4 r$-Factorization properties of regular kernel operators}

As explained in the introduction, the main purpose of this section is to shed further light into the relationship between factorization properties of the kind encountered in the previous section and the property of being a (regular) kernel operator. In particular, we wish to know to which extent such properties determine the kernel operators within the regular ones. Our main findings in this direction are collected in the next theorem.

Theorem 2 Let $X$ and $Y$ be Banach lattices and let $T \in \mathcal{L}^{r}(X, Y)$.

(i) Suppose both $X^{\prime}$ and $Y$ are order continuous, both have weak order units and there is a regular projection $P: Y^{\prime \prime} \rightarrow Y$. Under these assumptions, if $T \in$ $\mathcal{F}(X, Y)^{d d}$ then $T$-factors through a purely atomic Banach lattice. Moreover, if $T$ is positive then at least one of the factors (any one of them) can be chosen to be positive.

(ii) If $T$-factors through an order continuous purely atomic Banach lattice, then $T$ belongs to $\mathcal{F}(X, Y)^{d d}$.

(iii) Let $X$ be reflexive, let $Y$ be a $K B$ space and suppose both $X$ and $Y$ have weak order units. Then all the following are equivalent:

(a) $T$ is a kernel operator.

(b) $T r$-factors through a purely atomic Banach lattice $Z$ and the factors $S: X \rightarrow$ $Z$ and $R: Z \rightarrow Y$ can be chosen so that for every atom $z \in S(X)^{d d}, R z \neq 0$.

(c) $T r$-factors through a reflexive purely atomic Banach lattice.

Furthermore, in parts (b) and (c), if $T$ is positive then at least one of the factors (any one of them) can be chosen to be positive.

In proving the theorem, we shall make use of the following simple fact.

Lemma 2 Let $X$ and $Y$ be Banach lattices and let $T \in \mathcal{A}^{r}(X, Y)$. Then, for every $\varepsilon>0$, there are sequences $\left(f_{i}\right) \subset X_{+}^{\prime},\left(y_{i}\right) \subset Y_{+}$and $\left(\eta_{i}\right) \subset\{ \pm 1\}^{\mathbb{N}}$ so that $T=\sum_{i} \eta_{i} f_{i} \otimes y_{i}$ and $\sum_{i} f_{i}(x) g\left(y_{i}\right) \leq\left(2\|T\|_{r}+\varepsilon\right)\|x\|\|g\|\left(x \in X_{+}, g \in Y_{+}^{\prime}\right)$.

Proof Let $\varepsilon>0$ arbitrary, let $H$ be the family of all words in the digits 0 and 1 , and let $H_{n}:=\{h \in H:|h|=n\}(n \in \mathbb{N})$, where $|h|$ stands for the length of the word $h \in H$. Let $T \in \mathcal{A}^{r}(X, Y)$ arbitrary. We shall construct a family $\left\{T_{h}: h \in H\right\} \subset X_{+}^{\prime} \otimes Y_{+}$ as follows. First, let $e$ stand for the empty word and set $S_{e}:=T$. Then choose $T_{0}, T_{1} \in X_{+}^{\prime} \otimes Y_{+}$so that $\max \left\{\left\|\left(S_{e}\right)_{+}-T_{0}\right\|_{r},\left\|\left(S_{e}\right)_{-}-T_{1}\right\|_{r}\right\} \leq \varepsilon / 4$, and set $S_{0}:=\left(S_{e}\right)_{+}-T_{0}$ and $S_{1}:=\left(S_{e}\right)_{-}-T_{1}$. In general, if $T_{h}$ and $S_{h}$ are known, choose $T_{h 0}, T_{h 1} \in X_{+}^{\prime} \otimes Y_{+}$so that

$$
\max \left\{\left\|\left(S_{h}\right)_{+}-T_{h 0}\right\|_{r},\left\|\left(S_{h}\right)_{-}-T_{h 1}\right\|_{r}\right\} \leq \frac{\varepsilon}{4^{|h|+1}},
$$


and set $S_{h 0}:=\left(S_{h}\right)_{+}-T_{h 0}$ and $S_{h 1}:=\left(S_{h}\right)_{-}-T_{h 1}$.

Let $s(h)(h \in H)$ be the number of ones in the word $h$. It is then easy to see that

$$
\left\|T-\sum_{n=1}^{m} \sum_{h \in H_{n}}(-1)^{s(h)} T_{h}\right\|_{r}=\left\|\sum_{h \in H_{m}}(-1)^{s(h)} S_{h}\right\|_{r} \leq \frac{\varepsilon}{2^{m}} \quad(m \in \mathbb{N}) .
$$

Furthermore, since

$$
\sum_{n} \sum_{h \in H_{n}}\left\|T_{h}\right\|_{r} \leq 2\|T\|_{r}+\frac{\varepsilon}{2}+\sum_{n>1} 2^{n}\left(\frac{\varepsilon}{4^{n-1}}+\frac{\varepsilon}{4^{n}}\right) \leq 2\|T\|_{r}+5 \varepsilon
$$

convergence of $\sum_{h \in H}(-1)^{s(h)} T_{h}$ is absolute. For each $h \in H$, let $\left\{f_{i, h}: 1 \leq i \leq\right.$ $k(h)\} \subset X_{+}^{\prime}$ and $\left\{y_{i, h}: 1 \leq i \leq k(h)\right\} \subset Y_{+}$be so that $\sum_{i=1}^{k(h)} f_{i, h} \otimes y_{i, h}=T_{h}$. Then

$$
T=\sum_{h} \sum_{i=1}^{k(h)}(-1)^{s(h)} f_{i, h} \otimes y_{i, h}
$$

and since $0 \leq \sum_{i \in S} f_{i, h} \otimes y_{i, h} \leq T_{h}$ for every $S \subseteq\{1, \ldots, k(h)\}(h \in H)$, also the series $\sum_{h, i}(-1)^{s(h)} f_{i, h} \otimes y_{i, h}$ converges absolutely to $T$. Both claims of the lemma now follow easily from this last observation and (1) above.

Proof of Theorem 2 (i) It will suffice to consider the case where $T \geq 0$, for if $T \in$ $\mathcal{L}^{r}(X, Y)$ is such that $T_{+} r$-factors through a Banach lattice $Z_{1}$ with factors $R_{1} \in$ $\mathcal{L}^{r}\left(Z_{1}, Y\right)$ and $S_{1} \in \mathcal{L}^{r}\left(X, Z_{1}\right)$, and $T_{-} r$-factors through a Banach lattice $Z_{2}$ with factors $R_{2} \in \mathcal{L}^{r}\left(Z_{2}, Y\right)$ and $S_{2} \in \mathcal{L}^{r}\left(X, Z_{2}\right)$, then $T r$-factors through $Z_{1} \oplus_{2} Z_{2}$ with factors $R: Z_{1} \oplus_{2} Z_{2} \rightarrow Y,\left(z_{1}, z_{2}\right) \mapsto R_{1} z_{1}-R_{2} z_{2}$, and $S: X \rightarrow Z_{1} \oplus_{2} Z_{2}$, $x \mapsto\left(S_{1} x, S_{2} x\right)$ (here, $Z_{1} \oplus_{2} Z_{2}$ stands for the $\ell_{2}$-sum of $Z_{1}$ and $Z_{2}$, with the natural order).

So let $T \geq 0$ and let $\xi$ and $e$ be weak order units for $X^{\prime}$ and $Y$, respectively. Set $S_{n}:=T \wedge(n \xi \otimes e)(n \in \mathbb{N})$, and let $T_{n}:=S_{n+1}-S_{n}(n \in \mathbb{N})$. Note that since $X^{\prime}$ and $Y$ are order continuous, $S_{n} \in \mathcal{A}^{r}(X, Y)(n \in \mathbb{N})$. Clearly, $\{\xi \otimes e\}^{d d}=\mathcal{F}(X, Y)^{d d}=$ $\mathcal{A}^{r}(X, Y)^{d d}$, and so, for every $R \in \mathcal{A}^{r}(X, Y) \cap[0, T], R \wedge(n \xi \otimes e) \uparrow R$. It follows that $\sup _{n} S_{n} x \geq R x$ for every $R \in \mathcal{A}^{r}(X, Y) \cap[0, T]$ and $x \in X_{+}$, and hence, that $\sup _{n} S_{n} x \geq \sup \left\{R x: R \in \mathcal{A}^{r}(X, Y) \cap[0, T]\right\}=T x$. Since $Y$ is order continuous and $S_{n} x \uparrow T x\left(x \in X_{+}\right),\left(S_{n}\right)$ converges to $T$ in the strong operator topology.

Let $\varepsilon>0$ arbitrary. For each $n \in \mathbb{N}$, choose $\widetilde{T}_{n} \in X_{+}^{\prime} \otimes Y_{+}$so that $\| T_{n}-$ $\widetilde{T}_{n} \|_{r}<2^{-n} \varepsilon$, and choose sequences $\left(f_{i, n}\right)_{i=1}^{k(n)} \subset X_{+}^{\prime}$ and $\left(y_{i, n}\right)_{i=1}^{k(n)} \subset Y_{+}$such that $\widetilde{T}_{n}=\sum_{i=1}^{k(n)} f_{i, n} \otimes y_{i, n}$. Since $\sum_{n} T_{n} x=T x(x \in X)$, the series $\sum_{n} \widetilde{T}_{n}$ converges in the strong operator topology to some positive linear operator $\widetilde{T}$. Clearly, $\sum_{n}\left(T_{n}-\right.$ $\left.\widetilde{T}_{n}\right)=: T_{0} \in \mathcal{A}^{r}(X, Y)$, so, by the previous lemma, there are sequences $\left(f_{i, 0}\right) \subset X_{+}^{\prime}$, $\left(y_{i, 0}\right) \subset Y_{+}$and $\left(\eta_{i}\right) \subset\{ \pm 1\}^{\mathbb{N}}$ such that $T_{0}(x)=\sum_{i} \eta_{i} f_{i, 0}(x) y_{i, 0}$ and

$$
\sum_{i} f_{i, 0}(x) g\left(y_{i, 0}\right) \leq\left(2\left\|T_{0}\right\|_{r}+\varepsilon\right)\|x\|\|g\| \leq 3 \varepsilon\|x\|\|g\| \quad\left(x \in X_{+}, g \in Y_{+}^{\prime}\right) .
$$


One readily sees that $T x=\sum_{n \in \mathbb{N}} \sum_{i=1}^{k(n)} f_{i, n}(x) y_{i, n}+\sum_{i} \eta_{i} f_{i, 0}(x) y_{i, 0}(x \in X)$, and so, that there are sequences $\left(f_{i}\right) \subset X_{+}^{\prime},\left(y_{i}\right) \subset Y_{+}$and $\left(\tau_{i}\right) \subset\{ \pm 1\}^{\mathbb{N}}$ such that $T x=\sum_{i} \tau_{i} f_{i}(x) y_{i}(x \in X)$ and

$$
\begin{aligned}
\sum_{i} f_{i}(x) g\left(y_{i}\right) & \leq\left(3 \varepsilon+\|\widetilde{T}\|_{r}\right)\|x\|\|g\| \\
& \leq\left(\|T\|_{r}+4 \varepsilon\right)\|x\|\|g\| \quad\left(x \in X_{+}, g \in Y_{+}^{\prime}\right) .
\end{aligned}
$$

Now, let $\Gamma$ be the solid hull of the set $\left\{\left(f_{i}(x)\right): x \in X_{[1]}\right\} \subset \mathbb{R}^{\mathbb{N}}$, where $\mathbb{R}^{\mathbb{N}}$ is endowed with the canonical order, and let $Z_{0}:=\operatorname{sp}(\Gamma)$. Then $\Gamma$ is an absolutely convex, absorbent and radially bounded subset of $Z_{0}$. (That $\Gamma$ is absolutely convex is obvious; that it is absorbent follows on noting that if $\xi=\sum_{i=1}^{n} \alpha_{i} \xi_{i}(\xi \neq 0)$, with $\xi_{1}, \ldots, \xi_{n} \in \Gamma$, then $\left(\sum_{j=1}^{n}\left|\alpha_{j}\right|\right)^{-1}|\xi| \leq\left(\sum_{j=1}^{n}\left|\alpha_{j}\right|\right)^{-1} \sum_{i=1}^{n}\left|\alpha_{i}\right|\left|\xi_{i}\right| \in \Gamma$; to see $\Gamma$ is radially bounded simply note that for every $\xi \in Z_{0} \backslash\{0\}$, if $k$ is such that $\xi(k) \neq 0$, then $t \xi \in \Gamma \Rightarrow|t \xi(k)| \leq f_{k}(x)$ for some $x \in X_{[1]} \cap X_{+} \Rightarrow|t| \leq\left\|f_{k}\right\| /|\xi(k)|$.) The Minkowski functional of $\Gamma$ is thus a lattice norm on $Z_{0}$, and we shall define $Z$ as the completion of $Z_{0}$ with respect to such norm. Also note that, by (2),

$$
\sum_{i} \xi_{i} g\left(y_{i}\right) \leq 2\|T\|_{r}+8 \varepsilon \quad\left(\left(\xi_{i}\right) \in \Gamma, g \in Y_{[1]}^{\prime}\right)
$$

for $\left(\xi_{i}\right) \in \Gamma \Rightarrow\left(\left|\xi_{i}\right|\right) \leq\left(f_{i}(x)\right)$ for some $x \in X_{[1]} \cap X_{+}$, and $g \in Y_{[1]}^{\prime} \Rightarrow g_{-}, g_{+} \in$ $Y_{[1]}^{\prime}$.

Define $S: X \rightarrow Z$ by $S x:=\left(\tau_{i} f_{i}(x)\right)(x \in X)$, and $U: Y^{\prime} \rightarrow Z^{\prime}$ by $U g:=$ $\left(g\left(y_{i}\right)\right)\left(g \in Y^{\prime}\right)$. By the previous paragraph, it is clear that both $U$ and $S$ are welldefined. Furthermore, both are regular and therefore bounded (in fact, it is clear from the previous paragraph that their norms are bounded by $2\|T\|_{r}+8 \varepsilon$ and 1 , respectively). Also, for every $x \in X$ and $g \in Y^{\prime}$,

$$
g(T x)=\sum_{i} \tau_{i} f_{i}(x) g\left(y_{i}\right)=U g(S x)=\left(U^{\prime}(S x)\right)(g)
$$

so $T x=U^{\prime}(S x)(x \in X)$. Let $R:=\left.P \circ U^{\prime}\right|_{Z}$. Then $T=R S$ is a factorization of the kind claimed by the theorem. Note that if $z_{i}$ is the sequence (or atom) in $Z$ with 1 in the $i$-th entry and 0 in all the others, then $\left(U^{\prime} z_{i}\right)(g)=z_{i}(U g)=g\left(y_{i}\right)\left(g \in Y^{\prime}\right)$, i.e., $U^{\prime} z_{i}=y_{i}$, and therefore $R z_{i}=y_{i} \neq 0(i \in \mathbb{N})$.

As for the final assertion of part (i), simply note that above, we could have defined $S: X \rightarrow Z$ by $S x:=\left(f_{i}(x)\right)(x \in X)$, and $U: Y^{\prime} \rightarrow Z^{\prime}$ by $U g:=\left(\tau_{i} g\left(y_{i}\right)\right)\left(g \in Y^{\prime}\right)$.

(ii) Here, we could argue as in Corollary 1. Instead, though, we shall provide an alternative, more elementary and self-contained argument. To this end, suppose $T r$-factors through an atomic Banach lattice $Z$, i.e., there are $R \in \mathcal{L}^{r}(Z, Y)$ and $S \in \mathcal{L}^{r}(X, Z)$ so that $R S=T$. Consider first the case where $R$ and $S$ (and therefore $T$ ) are positive. Let $\left\{z_{i}: i \in I\right\}$ be the collection of all normalized atoms of $Z$ whose linear span, since $Z$ is order continuous, must be dense in $Z$. Let $\left\{z_{i}^{*}: i \in I\right\}$ be 
the corresponding set of biorthogonal functionals in $Z^{\prime}$, and define $f_{i}:=S^{\prime} z_{i}^{*}$ and $y_{i}:=R z_{i}(i \in I)$. Then

$$
T x=R S x=R\left(\sum_{i} z_{i}^{*}(S x) z_{i}\right)=\sum_{i} z_{i}^{*}(S x) R z_{i}=\sum_{i} f_{i}(x) y_{i} \quad(x \in X) .
$$

We thus have that $0 \leq \sum_{i \in F} f_{i} \otimes y_{i} \uparrow T$, where $F$ runs over all finite subsets of $I$, and hence, $T \in \mathcal{F}(X, Y)^{d d}$.

In the general case, $T=S_{+} R_{+}+S_{-} R_{-}-S_{+} R_{-}-S_{-} R_{+}$, and by the previous discussion, $S_{+} R_{+}, S_{-} R_{-}, S_{+} R_{-}$and $S_{-} R_{+}$belong to $\mathcal{F}(X, Y)^{d d}$.

(iii) (a) $\Rightarrow$ (b): Let $X$ and $Y$ be as in the hypotheses and let $T: X \rightarrow Y$ be a regular kernel operator. Since $X$ is order continuous and has a weak order unit, $X^{\prime}$ has a weak order unit too (see for instance [10, Theorem 1.b.14]); and since $Y$ is a KB space, $Y$ is a band in $Y^{\prime \prime}$. Thus, arguing as in the proof of part (i), one can construct a purely atomic Banach lattice $Z$ and operators $R \in \mathcal{L}^{r}(Z, Y)$ and $S \in \mathcal{L}^{r}(X, Z)$, such that $R S=T, S(X)^{d d}=Z$ and $R z \neq 0$ for every atom $z \in Z$.

It is also clear, from the proof of part (i), that if $T \geq 0$, at least one of the two factors (any one of them) can be chosen to be positive.

(b) $\Rightarrow$ (c): Let $Z$ be a purely atomic Banach lattice such that there is an $r$ factorization of $T$ through $Z$ with factors $R$ and $S$ as in (iii)(b). Let $S_{1} \in \mathcal{L}^{r}(X, Z)_{+}$ and $R_{1} \in \mathcal{L}^{r}(Z, Y)_{+}$be such that $\pm S \leq S_{1}$ and $\pm R \leq R_{1}$. What follows now goes more or less along the lines of the argument of the proof of [2, Theorem 5.45], which under the given assumptions, yields an $r$-factorization of $T$ through a reflexive purely atomic Banach lattice. Before starting with the argument, and for the reader's convenience, we recall a few facts.

Given a Banach lattice $E$ and a convex solid norm-bounded subset of it, $W$ say, we write $E_{W}$ for the Banach lattice whose underlying vector space is $\{x \in E$ : $\left.\sum_{i=1}^{\infty}\|x\|_{i}^{2}<\infty\right\}$ (where for each $i \in \mathbb{N},\|\cdot\|_{i}$ is the (equivalent) norm on $E$ given by the Minkowski functional of the set $\left.2^{i} W+2^{-i} E_{[1]}\right)$, endowed with the norm $\|x\|:=\left(\sum_{i=1}^{\infty}\|x\|_{i}^{2}\right)^{\frac{1}{2}}\left(x \in E_{W}\right)$. From [8, Lemma 1], we know that $W \subseteq\left(E_{W}\right)_{[1]}$ and that if $W$ is relatively weakly compact then $E_{W}$ is reflexive. Furthermore, $E_{W}$ is a Banach lattice and an order ideal of $E$ such that the natural inclusion $\imath: E_{W} \rightarrow E$ and its adjoint $\iota^{\prime}: E^{\prime} \rightarrow E_{W}^{\prime}$ are both lattice homomorphisms ([2, Theorem 5.41]). Lastly, if $W$ is the convex solid hull of a relatively weakly compact set, then $E_{W}^{\prime}$ is order continuous, and therefore, a KB space ([2, Theorem 5.43]).

We now go through the argument. Consider first the weakly compact operator $S$. Let $U$ be the solid hull of $S_{1}\left(X_{[1]}\right)$ in $Z$ and let $\Psi:=Z_{U}$ (so $S\left(X_{[1]}\right) \cup S_{1}\left(X_{[1]}\right) \subseteq$ $\left.U \subseteq \Psi_{[1]}\right)$. Then $\Psi$ is a Banach lattice and an order ideal of $\underset{\widetilde{S}}{Z}$, hence purely atomic, whose dual $\Psi^{\prime}$ is a KB space. Let $\widetilde{S}: X \rightarrow \Psi, x \mapsto S x$, let $\widetilde{S}_{1}: X \rightarrow \Psi, x \mapsto S_{1} x$, and let $l: \Psi \rightarrow Z$ be the natural inclusion (which is a lattice homomorphism). Then $\pm \widetilde{S} \leq \widetilde{S}_{1}$, so $\widetilde{S}$ is regular, and clearly, $S=\imath \circ \widetilde{S}$.

Consider next the map $L:=R \circ \imath: \Psi \rightarrow Y$. Let $L_{1}:=R_{1} \circ \iota: \Psi \rightarrow Y$. Since $\Psi^{\prime}$ is order continuous and $Y$ is a KB space, both $L$ and $L_{1}$ are weakly compact ([2, Theorem 5.27]). Thus, $L_{1}^{\prime}$ is weakly compact, and since $\Psi^{\prime}$ is a KB space, the solid hull of $L_{1}^{\prime}\left(Y_{[1]}^{\prime}\right), V$ say, is relatively weakly compact ([2, Theorem 4.39(2)]). 
Let $\Upsilon:=\left(\Psi^{\prime}\right)_{V}$. Then $\Upsilon$ is a reflexive Banach lattice and the natural inclusion $\jmath: \Upsilon \rightarrow \Psi^{\prime}$ and its adjoint $J^{\prime}: \Psi^{\prime \prime} \rightarrow \Upsilon^{\prime}$ are both lattice homomorphisms. Like above, note that $L^{\prime}\left(Y_{[1]}^{\prime}\right) \cup L_{1}^{\prime}\left(Y_{[1]}^{\prime}\right) \subseteq V \subseteq \Upsilon_{[1]}$. So $H: Y^{\prime} \rightarrow \Upsilon, y^{\prime} \mapsto L^{\prime} y^{\prime}$, and $H_{1}: Y^{\prime} \rightarrow \Upsilon, y^{\prime} \mapsto L_{1}^{\prime} y^{\prime}$, are well-defined. Furthermore, $L^{\prime}=j \circ H$ and $\pm H \leq H_{1}$.

Let $\Phi$ be the closure of $J^{\prime}(\Psi)$ in $\Upsilon^{\prime}$. Clearly $\Phi$ is reflexive and we will show next it is also atomic. For this, let $\left\{e_{i}: i \in I\right\}$ be the set of all normalized atoms of $\Psi$, let $\gamma_{i}:=J^{\prime}\left(e_{i}\right)(i \in I)$ and let $\Gamma:=\left\{\gamma_{i}: i \in I\right\}$. Note that $0 \notin \Gamma$, for $H^{\prime}\left(\gamma_{i}\right)=L^{\prime \prime}\left(e_{i}\right)=L\left(e_{i}\right)=R\left(e_{i}\right) \neq 0(i \in I)$, where we have identified $\Psi$ with $\imath(\Psi)$. Furthermore, each $\gamma_{i}$ is an atom in $\Phi$. To see it, fix $i$ and let $\phi \in \Phi$ be such that $0<\phi \leq \gamma_{i}$. Let $\left(\psi_{n}\right) \subset \Psi_{+}$be such that $\phi=\lim _{n} J^{\prime}\left(\psi_{n}\right)$. Without loss of generality, we can assume $0<J^{\prime}\left(\psi_{n} \wedge e_{i}\right) \leq \gamma_{i}(n \in \mathbb{N})$. Since $X$ is atomic $\psi_{n} \wedge e_{i}$ is a supremum of elements of the form $\sum_{j \in F} t_{j} e_{j}$, with $F \subset I$ finite and $t_{j} \geq 0, j \in F$. But $0 \leq \sum_{j \in F} t_{j} e_{j} \leq \psi_{n} \wedge e_{i} \Rightarrow 0 \leq \sum_{j \in F} t_{j} \gamma_{j} \leq \gamma_{i} \Rightarrow t_{j}=0(j \in F \backslash\{i\})$. Thus, $\psi_{n} \wedge e_{i} \in \mathbb{R} e_{i}(n \in \mathbb{N})$, and in turn, $\phi \in \mathbb{R} \gamma_{i}$. It remains to show $\Gamma^{d d}=\Phi$. Clearly, it will suffice to show $\Gamma^{d}=\{0\}$. For this, suppose towards a contradiction there is a $b \in \Gamma^{d} \backslash\{0\}$. Since $\Gamma^{d}$ is a band, we can further assume, by replacing $b$ if necessary, that $0<b=J^{\prime}(\psi)$ for some $\psi \in \Psi_{+}$. Since $\Psi$ is atomic, we would have that $0<t e_{i} \leq \psi$ for some $t>0$ and $i \in I$, and hence, that $\gamma_{i}=\gamma_{i} \wedge t^{-1} b=0$, which is clearly impossible. Thus, $\Phi$ is atomic.

Finally, letting $P: Y^{\prime \prime} \rightarrow Y$ be the corresponding band projection, and noting that $L^{\prime \prime}=H^{\prime} \circ J^{\prime}$ and that $L^{\prime \prime}\left(\Psi^{\prime \prime}\right) \subseteq Y$, it is easy to see that the maps $\left.J^{\prime}\right|_{\Psi} ^{\Phi}$ and $\left.P \circ H^{\prime}\right|_{\Phi}$ provide a factorization of $L$ through $\Phi$ with regular factors. In turn, $\left.J^{\prime}\right|_{\Psi} ^{\Phi} \circ \widetilde{S}$ and $\left.P \circ H^{\prime}\right|_{\Phi}$ provide the desired factorization of $T$ through $\Phi$.

If $T \geq 0$, then we can assume $R$ (resp. $S$ ) to be positive. If $R$ (resp. $S$ ) is positive it should be clear from the above argument that the same will be true about $\left.P \circ H^{\prime}\right|_{\Phi}$ $\left(\right.$ resp. $\left.\left.J^{\prime}\right|_{\Psi} ^{\Phi} \circ \tilde{S}\right)$.

(c) $\Rightarrow$ (a): This is immediate from (ii).

Remark 5 It should be pointed out that it is not possible, in general, for a positive $T$ in parts (i) and (iii) of the theorem, to produce an $r$-factorization with both factors positive (see the example at the end of [15]). This appears to be the main obstacle in extending parts (i) and (iii) to arbitrary non-separable Banach lattices. It is not hard to see (from the proof of the theorem), though, that if $T \geq 0$ then for every $\varepsilon>0$ there exist an atomic Banach lattice $Z$ and operators $R \in \mathcal{L}^{r}(Z, Y)_{+}$(resp. $R \in$ $\left.\mathcal{L}^{r}(Z, Y)_{+}+\mathcal{A}^{r}(Z, Y)_{[\varepsilon]}\right)$ and $S \in \mathcal{L}^{r}(X, Z)_{+}+\mathcal{A}^{r}(X, Z)_{[\varepsilon]}\left(\right.$ resp. $\left.S \in \mathcal{L}^{r}(X, Z)_{+}\right)$ such that $R S=T$.

It follows easily from Theorem 2 that for every reflexive Banach lattice $X$ with a weak order unit there is a reflexive purely atomic Banach lattice $Z$ such that $\mathcal{I}^{r}(X)=$ $\left\{T \in \mathcal{L}^{r}(X): T r\right.$-factors through $\left.Z\right\}$. Information on the lattice of ideals of $\mathcal{L}^{r}(Z)$ may then lead to information on that of $\mathcal{I}^{r}(X)$ via the map that associates to every ideal $\mathcal{J}$ of $\mathcal{L}^{r}(Z)$ the ideal $\left\{T \in \mathcal{L}^{r}(X): \pm T \leq S \in \operatorname{sp}\left(\mathcal{L}^{r}(Z, X) \circ \mathcal{J} \circ \mathcal{L}^{r}(X, Z)\right)\right\}$ of $\mathcal{I}^{r}(X)$. Of course, the usefulness of this approach, for a given $X$, will depend on the properties of the latter map, and in turn, on our choice of $Z$. 
Open Access This article is licensed under a Creative Commons Attribution 4.0 International License, which permits use, sharing, adaptation, distribution and reproduction in any medium or format, as long as you give appropriate credit to the original author(s) and the source, provide a link to the Creative Commons licence, and indicate if changes were made. The images or other third party material in this article are included in the article's Creative Commons licence, unless indicated otherwise in a credit line to the material. If material is not included in the article's Creative Commons licence and your intended use is not permitted by statutory regulation or exceeds the permitted use, you will need to obtain permission directly from the copyright holder. To view a copy of this licence, visit http://creativecommons.org/licenses/by/4.0/.

\section{References}

1. Abramovich, Y.A., Aliprantis, C.D.: Positive Operators. Handbook of the Geometry of Banach Spaces I, pp. 85-122. North-Holland, Amsterdam (2001)

2. Aliprantis, C.D., Burkinshaw, O.: Positive Operators. Pure and Applied Mathematics, p. 119. Academic Press, Orlando (1985)

3. Arendt, W., Sourour, A.R.: Ideals of regular operators on $\ell_{2}$. Proc. Am. Math. Soc. 88(1), 93-96 (1983)

4. Arendt, W., Schwarz, H.-U.: Ideale regulärer Operatoren und Kompaktheit positiver Operatoren zwischen Banachverbänden. Math. Nachr. 131, 7-18 (1987)

5. Beauzamy, B.: Introduction to Banach Spaces and Their Geometry. In: Nachbin, L. (ed.) North-Holland Mathematics Studies, p. 68. North-Holland Publishing Co., Amsterdam (1982)

6. Diestel J.: Sequences and series in Banach spaces. In: Graduate Texts in Mathematics, p. 92. Springer, New York (1984)

7. Dodds, P.G., Fremlin, D.H.: Compact operators in Banach lattices. Israel J. Math. 34(4), 287-320 (1979)

8. Davis, W.J., Figiel, T., Johnson, W.B., Pelczynski, A.: Factoring weakly compact operators. J. Funct. Anal. 17, 311-327 (1974)

9. Diestel, J., Uhl, J.J.: Vector Measures. Mathematical Surveys, p. 15. American Mathematical Society, Providence (1977)

10. Lindenstrauss, J., Tzafriri, L.: Results in Mathematics and Related Areas. Classical Banach Spaces II: Function Spaces, p. 97. Springer, Berlin (1979)

11. Lewis, D.R., Stegall, C.: Banach spaces whose duals are isomorphic to $l_{1}(\Gamma)$. J. Funct. Anal. 12, 177-187 (1973)

12. Maurey, B.: Théorèmes de factorisation pour les opérateurs linéaires à valeurs dans les espaces $L^{p}$, Astérisque, p. 11. Société Mathématique de France, Paris (1974)

13. Meyer-Nieberg, P.: Banach Lattices. Universitext. Springer, Berlin (1991)

14. Oikhberg, T.: Reverse monotone approximation property: function spaces in modern analysis. Contemp. Math. Am. Math. Soc 547, 197-206 (2011)

15. Popovici, I.M., Vuza, D.T.: Factoring compact operators and approximable operators. Z. Anal. Anwend. 9(3), 221-233 (1990)

16. Schaefer, H.H.: Banach Lattices and Positive Operators. Springer, Berlin (1974)

17. Schep, A.R., Wolff, M.: Semicompact operators. Indag. Math. (N.S.) 1(1), 115-125 (1990)

18. Schachermayer, W., Weis, L.: Almost compactness and decomposability of integral operators. Proc. Am. Math. Soc. 81(4), 595-599 (1981)

19. Weis, L.W.: Banach lattices with the subsequence splitting property. Proc. Am. Math. Soc. 105(1), 87-96 (1989)

20. Wickstead, A.W.: Positive compact operators on Banach lattices: some loose ends. Positivity 4(3), 313-325 (2000)

21. Wojtaszczyk, P.: Cambridge Studies in Advanced Mathematics. Banach Spaces for Analysts, p. 25. Cambridge University Press, Cambridge (1991)

22. Zaanen, A.C.: Riesz Spaces II, p. 30. North-Holland Mathematical Library, North-Holland, Amsterdam (1983)

Publisher's Note Springer Nature remains neutral with regard to jurisdictional claims in published maps and institutional affiliations. 